\title{
A new method for taming tensor sum-integrals
}

\author{
Ioan Ghișoiu and York Schröder \\ Faculty of Physics, University of Bielefeld, 33501 Bielefeld, Germany \\ E-mail: ighisoiu@physik.uni-bielefeld.de, yorks@physik.uni-bielefeld.de
}

\begin{abstract}
We report on the computation of a class of massless bosonic three-loop vacuum sum-integrals which are key building blocks for an evaluation of the Debye screening mass in hot QCD. Generalizing known techniques and introducing the concept of tensor reduction by dimensionality shifts (known to the zero-temperature community since the work of Tarasov in 1996) to finite temperature, we are able to treat hitherto unaccessible cases, which will allow us to finalize the long-term project of NNLO Debye mass evaluation.
\end{abstract}




\section{Contents}

1 Introduction $\quad 1$

2 Decomposition of $\mathcal{M}_{N,-2} \quad 3$

2.1 Splitting off trivial parts 4

2.2 Taming the tensors 5

2.3 Decomposition of spectacles-type sum-integrals 6

3 Non-zero modes $\quad 7$

3.1 Generic formulae for 2-point subtraction terms $\quad 7$

3.2 Generic formulae for divergences $V^{\mathrm{d}}$ of non-zero modes 8

3.3 Specific results for divergences $V^{\mathrm{d}}$ of non-zero modes 9

3.4 Finite parts $V^{\mathrm{f}}$ of non-zero modes 9

4 Zero modes $\quad 9$

4.1 Generic results for divergences $S^{\text {d }}$ of zero modes 10

4.2 Specific results for divergences $V^{\mathrm{z}, \mathrm{d}}$ of zero modes 10

4.3 Finite parts $V^{z, f}$ of zero modes 11

5 Result for $\mathcal{M}_{3,-2} \quad 11$

6 Conclusions $\quad 12$

$\begin{array}{ll}\text { A Standard integrals } & 13\end{array}$

B IBP relations for 2-loop sum-integrals 13

$\begin{array}{ll}\text { C IBP relations for 3-loop zero modes } & 14\end{array}$

$\begin{array}{ll}\text { D Numerical evaluation of finite integrals } & 15\end{array}$

$\begin{array}{lll}\text { E Cross-checks } & 23\end{array}$

\section{Introduction}

Taking as a motivation the startling imbalance between highly developed analytic, systematic algorithmic as well as numeric methods for multi-loop integrals in zero-temperature field theory on the one hand (see, e.g. [1]), and the many unsolved technical challenges one is 
confronted with when dealing with phenomenological problems in cosmological or heavy-ioncollision related contexts that are formulated within finite temperature field theory on the other hand (see, e.g. [2-6]), we continue our line of systematic investigation of the latter class of problems [7-12]. Once more diving into a complicated and rather technical issue, our aim is to exhibit and develop generic tools that allow for progress on the thermodynamic (equilibrium) front. Nevertheless, while in this note we merely focus on a specific class of multi-loop sum-integral that arise in the thermodynamic setting, our final result will find a concrete application in the determination of matching parameters (such as the Debye screening mass) in the systematic program of an effective theory treatment of hot QCD thermodynamics $[13,14]$. We relegate the finalization of the long-term project of determining the Debye screening mass $m_{\mathrm{E}}^{2}$ of hot Yang-Mills theory to NNLO [9] to an upcoming publication [15], and here focus on the well-separated problem of evaluating the last missing sum-integral in dimensional regularization, up to its constant term. Let us just mention here that, once full 3-loop results for $m_{\mathrm{E}}^{2}$ (interpreted as a matching coefficient between $4 \mathrm{~d}$ thermal QCD and $3 \mathrm{~d}$ electrostatic QCD (EQCD) in the framework of dimensionally reduced effective theories [13, 14]) are available, a certain contribution of order $g^{7}$ to the hot QCD pressure can be deduced, and refer to Sec. 6 of Ref. [9] for a more detailed discussion of this potential phenomenological application.

There are a number of well-established techniques that prove useful when evaluating sum-integrals at higher loop order. Starting from the seminal work of Arnold and Zhai [2], a number of specific cases have appeared in several applications, ranging from 3-loop sumintegrals needed for QCD thermodynamics [2,3], to even some 4-loop cases that have served to quantify high-order corrections in the thermodynamics of scalar theory [16, 17] as well as large $N_{\mathrm{f}}$ QCD [18]. In each case, the corresponding sum-integrals that appear in the calculations have been evaluated to sufficient depth in their $\epsilon$-expansions, building a (small but valuable) database of master sum-integrals. There is an urgent need to enlarge this database, e.g. in order to make progress on the precision of matching computations [9] (involving moments of 3-loop self-energies) or on the determination of the physical leading order (for a justification of this term see e.g. Sec. 6 of [9]) of hot QCD observables [4, 10] (where further 4-loop masters are required).

More recently, in a series of works we have re-examined these computational techniques as well as most of the known specific cases of sum-integrals, and put them on a more generic footing, using notation that allows for generalizations $[8,9,11,12]$. Performing those generalizations for computing previously unknown cases [12], we have pointed out that integrationby-parts (IBP) relations can be used profitably here as well. IBP relations have already been successfully applied in the thermal context to the reduction process itself $[9,10]$, but now they can also be employed to help in evaluating the corresponding master sum-integrals that remain after the reduction algorithm has halted - mainly in order to transform infrared divergent parts of dimensionally regularized masters in terms of convergent ones [12]. We will see that also in the present context, they play an important role.

In this note, we wish to infuse another new idea to the art of evaluating sum-integrals, 
namely to use the ideas of Tarasov [19] for tensor reduction. The main goal is to avoid the traditional approach of projection methods that - if applied to thermal field theory where the rest frame of the heat bath breaks rotational invariance and introduces a vector $U=(1,(0))$ that all tensors can depend on - lead to inverse structures (such as $1 / \mathbf{p}^{2}$ ) of a different form than propagators, hence leading outside the class of sum-integrals that one has started with (in fact even outside its natural generalization as suggested by IBP [10, 11]). The price to pay is a shift (in units of two) of the dimensionality of the integral measure, and an increase of powers of the propagators that are present in the corresponding sum-integral [19]. We will argue that this price is not too high, and show explicitly that one can deal with these dimensionally shifted cases.

As a concrete example on which to test our new methods, we will consider a specific massless bosonic three-loop sum-integral of mass dimension two (which we shall name $\mathcal{M}_{3,-2}$ ) here, which constitutes one of the last building blocks needed for evaluating the Debye screening mass of thermal Yang-Mills theory. In fact, in order to stress the generality of our method, we will treat a more general (infinite) class of sum-integrals $\left(\mathcal{M}_{N,-2}\right.$, for integer values of $\left.N\right)$ for most part of the paper, of which $\mathcal{M}_{3,-2}$ is just a special case.

The paper is organized as follows. In Sec. 2, we introduce the class of 3-loop tensor sum integrals $\mathcal{M}_{N,-2}$ on which we wish to test our new idea of tensor reduction, and explain its decomposition into scalar pieces. Owing to the structure of the latter (they all contain two one-loop sub-integrals and are hence of so-called spectacles-type) we further decompose them into pieces that either allow for analytic solutions, or are finite such that they can be evaluated numerically. Sections 3 and 4 then deal with the evaluation of non-zero (Matsubara) modes and zero-modes in turn, specializing to the specific sum-integral $\mathcal{M}_{3,-2}$ for concrete results, which are then summarized in Sec. 5. We conclude in Sec. 6 and have relegated some technical material to the appendices.

\section{Decomposition of $\mathcal{M}_{N,-2}$}

The sum-integrals $\mathcal{M}_{N,-2}$ that we shall be concerned with here can be represented as a subset of a more general class as defined by (see Eqs. (A.23), (A.30) of Ref. [3] and the review [11])

$$
\hat{i}_{i} \equiv \mathcal{M}_{i, j} \equiv \mathcal{F}_{P Q R} \frac{\left[(Q-R)^{2}\right]^{-j}}{\left[P^{2}\right]^{i}} \frac{1}{Q^{2}(Q+P)^{2}} \frac{1}{R^{2}(R+P)^{2}} \text {. }
$$

We employ (Euclidean, bosonic) four-momenta $P=\left(P_{0}, \mathbf{p}\right)=\left(2 \pi n_{p} T, \mathbf{p}\right)$ with $P^{2}=P_{0}^{2}+\mathbf{p}^{2}$; the temperature of the thermal system is $T$; and the sum-integral symbol stands for

$$
\oiint_{P} \equiv T \sum_{n_{p} \in \mathbb{Z}} \int \frac{\mathrm{d}^{d} \mathbf{p}}{(2 \pi)^{d}}, \quad \text { with } d=3-2 \epsilon .
$$

The known non-trivial instances of the class Eq. (2.1) are $\mathcal{M}_{0,0}$ and $\mathcal{M}_{2,-2}$ [2] of (mass-) dimension four, as well as the dimension two case $\mathcal{M}_{1,0}$ [20], all of which have been reevaluated in unified notation in [11]. Furthermore, as has already been pointed out in the 
latter reference, the numerators of $\mathcal{M}_{N,-1}$ can be eliminated by using the denominator's invariance under momentum shifts $Q \rightarrow-P-Q$ and $R \rightarrow-P-R$, resulting in

$$
\mathcal{M}_{N,-1}=2 I_{1} \oiint_{P Q} \frac{1}{\left[P^{2}\right]^{N} Q^{2}(P+Q)^{2}}-\frac{1}{2} \mathcal{M}_{N-1,0},
$$

where the first term on the right-hand side (rhs) has factorized into a trivial 1-loop tadpole $I_{1}$ (cf. Eq. (A.2)) times a two-loop sum-integral of sunset-type which can be further factorized into a product of one-loop cases by IBP (see e.g. App. B) and is hence trivial as well, while the second term on the rhs is again in the class of Eq. (2.1), although without scalar products in the numerator and hence much simpler.

The massless bosonic 3-loop vacuum sum-integral $\mathcal{M}_{N,-2}$ is therefore defined as

$$
\mathcal{M}_{N,-2} \equiv \sum_{P Q R} \frac{\left[(Q-R)^{2}\right]^{2}}{\left[P^{2}\right]^{N} Q^{2} R^{2}(P+Q)^{2}(P+R)^{2}} .
$$

As mentioned above, one representative of this class, $\mathcal{M}_{2,-2}$, is already known $[2,3]$, since it has entered the 3-loop result for the thermodynamic pressure of hot QCD. However, its determination (as reviewed in [11]) was somewhat contrived, mainly due to difficulties in treating its numerator structure - a problem that has triggered much of what we report here, and that we will alleviate Sec. 2.2 below.

\section{$2.1 \quad$ Splitting off trivial parts}

Expanding the numerator of the class of massless bosonic 3-loop vacuum sum-integrals $\mathcal{M}_{N,-2}$ in Eq. $(2.4)$ as $\left[(Q-R)^{2}\right]^{2}=4(Q R)^{2}-4(Q R)\left(Q^{2}+R^{2}\right)+Q^{4}+R^{4}+2 Q^{2} R^{2}$, its spectacles-type structure becomes explicit when using the $\{$ scalar, vector,tensor $\}$-like 2-point functions

$$
\left\{\Pi_{a b}(P), \Pi_{a b}^{\mu}(P), \Pi_{a b}^{\mu \nu}(P)\right\} \equiv \sum_{Q} \frac{\left\{1, Q^{\mu}, Q^{\mu} Q^{\nu}\right\}}{\left[Q^{2}\right]^{a}\left[(P+Q)^{2}\right]^{b}},
$$

such that (omitting the argument $(P)$ of all functions $\Pi$ for brevity from now on)

$$
\begin{aligned}
\mathcal{M}_{N,-2} & =\sum_{P} \frac{1}{\left[P^{2}\right]^{N}}\left\{4 \Pi_{11}^{\mu \nu} \Pi_{11}^{\mu \nu}-4 \Pi_{01}^{\mu} \Pi_{11}^{\mu}-4 \Pi_{11}^{\mu} \Pi_{01}^{\mu}+\Pi_{-11} \Pi_{11}+\Pi_{11} \Pi_{-11}+2 \Pi_{01} \Pi_{01}\right\} \\
& =\sum_{P} \frac{1}{\left[P^{2}\right]^{N}}\left\{4 \Pi_{11}^{\mu \nu} \Pi_{11}^{\mu \nu}-2 P^{2} \Pi_{11} \Pi_{10}+2 \Pi_{10} \Pi_{10}\right\} \\
& =4 \sum_{P} \frac{1}{\left[P^{2}\right]^{N}} \Pi_{11}^{\mu \nu} \Pi_{11}^{\mu \nu}-2 I_{1}^{0} \oint_{P} \frac{\Pi_{11}}{\left[P^{2}\right]^{N-1}}+2 I_{N}^{0} I_{1}^{0} I_{1}^{0}
\end{aligned}
$$

where in the second and third line we have used some trivial properties of the scalar and vector-like 2-point-functions, which derive from the shifts $Q \rightarrow-P-Q$ or $Q \rightarrow-Q$ and read

$$
\begin{gathered}
\Pi_{a b}=\Pi_{b a}, \Pi_{a 0}=I_{a}^{0}, \Pi_{00}=I_{0}^{0}=\mathcal{F}_{Q} 1=0, \Pi_{-1 a}=P^{2} I_{a}^{0}+I_{a-1}^{0} ; \\
\Pi_{a b}^{\mu}=-P^{\mu} \Pi_{b a}-\Pi_{b a}^{\mu}, \Pi_{a a}^{\mu}=-\frac{P^{\mu}}{2} \Pi_{a a}, \Pi_{a 0}^{\mu}=0, \Pi_{0 a}^{\mu}=-P^{\mu} I_{a}^{0} .
\end{gathered}
$$


In Eq. (2.7), the third term is a product of 1-loop sum-integrals and hence known analytically, cf. Eq. (A.2), the second term is a 2-loop problem and hence equally trivial (it factorizes into 1-loop integrals via IBP, see Eq. (B.6)), while the first term needs further treatment and will be the focus of the next section.

\subsection{Taming the tensors}

To proceed, rewrite $\Pi_{11}^{\mu \nu} \Pi_{11}^{\mu \nu}=\Pi_{11}^{00} \Pi_{11}^{00}+2 \Pi_{11}^{0 i} \Pi_{11}^{0 i}+\Pi_{11}^{i j} \Pi_{11}^{i j}$. Noting that standard tensor reduction of the 3 -vectors present in the numerator of e.g. $\Pi_{11}^{0 i}=\frac{p^{0} p^{i}}{2 \mathbf{p}^{2}}\left\{I_{1}^{0}+\frac{P^{2}}{2} \Pi_{11}-2 \Pi_{11}^{00}\right\}$ produces inverses such as $1 / \mathbf{p}^{2}$, which we want to avoid since they are not contained in the structure of the original sum-integrals we started with, we choose to employ Tarasov's $T$-operator technique [19] to trade scalar products of 3 -vectors for higher dimensions.

To this end, we regard the (massless, 4d) sum-integrals as sums over corresponding (massive, 3d) spatial integrals, taken at specific values of the masses. In the spirit of Ref. [19], it is then advantageous to generate the irreducible scalar products of 3 -vectors (qr in our case) by derivatives acting on a generating function $\exp (-2 \alpha \mathbf{q r})$ :

$$
\begin{aligned}
& \mathcal{F}_{P} \frac{1}{\left[P^{2}\right]^{N}} \Pi_{11}^{0 i} \Pi_{11}^{0 i}=\left.T^{3} \sum_{P_{0} Q_{0} R_{0}} Q_{0} R_{0} \partial_{-2 \alpha} I_{N 1111}^{(3-2 \epsilon)}\left(P_{0}, Q_{0}, R_{0}, P_{0}+Q_{0}, P_{0}+R_{0} ; \alpha\right)\right|_{\alpha=0}, \\
& \mathcal{F}_{P} \frac{1}{\left[P^{2}\right]^{N}} \Pi_{11}^{i j} \Pi_{11}^{i j}=\left.T^{3} \sum_{P_{0} Q_{0} R_{0}} \partial_{-2 \alpha}^{2} I_{N 1111}^{(3-2 \epsilon)}\left(P_{0}, Q_{0}, R_{0}, P_{0}+Q_{0}, P_{0}+R_{0} ; \alpha\right)\right|_{\alpha=0},
\end{aligned}
$$

with the generic (massive, 3d) 3-loop vacuum integral

$$
I_{\nu_{1} \ldots 5}^{(d)}\left(m_{1 \ldots 5} ; \alpha\right) \equiv \int_{\mathbf{p q r}}^{(d)} \frac{e^{-2 \alpha(\mathbf{q r})}}{\left[\mathbf{p}^{2}+m_{1}^{2}\right]^{\nu_{1}}\left[\mathbf{q}^{2}+m_{2}^{2}\right]^{\nu_{2}}\left[\mathbf{r}^{2}+m_{3}^{2}\right]^{\nu_{3}}\left[(\mathbf{p}+\mathbf{q})^{2}+m_{4}^{2}\right]^{\nu_{4}}\left[(\mathbf{p}+\mathbf{r})^{2}+m_{5}^{2}\right]^{\nu_{5}}} .
$$

This integral has another useful representation, whose $\alpha$-derivatives will become simple: introducing Feynman parameters for the propagators $1 / A^{\nu}=\int_{0}^{\infty} \mathrm{d} \alpha \alpha^{\nu-1} e^{-\alpha A} / \Gamma(\nu)$, completing squares in the exponential, shifting momenta and rescaling them, it follows that

$$
I_{\nu_{1 \ldots 5}}^{(d)}=\left[\int_{\mathbf{p}}^{(d)} e^{-\mathbf{p}^{2}}\right]^{3}\left(\prod_{i=1}^{5} \int_{0}^{\infty} \mathrm{d} \alpha_{i} \frac{\alpha_{i}^{\nu_{i}-1} e^{-\alpha_{i} m_{i}^{2}}}{\Gamma\left(\nu_{i}\right)}\right)\left[D\left(\alpha_{j}\right)+\alpha 2 \alpha_{4} \alpha_{5}-\alpha^{2}\left(\alpha_{1}+\alpha_{4}+\alpha_{5}\right)\right]^{-d / 2}
$$

where $D\left(\alpha_{j}\right)$ is the graph polynomial [21]

$$
D\left(\alpha_{j}\right)=\left(\alpha_{2}+\alpha_{4}\right) \alpha_{3} \alpha_{5}+\left(\alpha_{3}+\alpha_{5}\right) \alpha_{2} \alpha_{4}+\alpha_{1}\left(\alpha_{2}+\alpha_{4}\right)\left(\alpha_{3}+\alpha_{5}\right) .
$$

The Gauss-integral in Eq. (2.12) depends on the chosen integral measure; in ours, it reads $\left[\int \frac{\mathrm{d}^{d} p}{(2 \pi)^{d}} e^{-\mathbf{p}^{2}}\right]^{3}=(4 \pi)^{-3 d / 2}$. Now, note that $\alpha$-derivatives acting on Eq. (2.12) lower the power of the last term by an integer $n$ (which can be interpreted as a shift $d \rightarrow d+2 n$ ), while producing additional polynomial pre-factors in the Feynman parameters $\alpha_{j}$. The latter 
can then be pulled out of the integral letting $\alpha_{j} \rightarrow \partial_{-m_{j}^{2}}$, and finally be absorbed in positive shifts of the propagator powers $\nu_{i}$. We need the two instances $(d=3-2 \epsilon)$

$$
\begin{aligned}
\left.\partial_{-2 \alpha} I_{N 1111}^{(d)}\right|_{\alpha=0} & =\left.\frac{d}{2} \partial_{-m_{4}^{2}} \partial_{-m_{5}^{2}} \mathcal{D}^{+} I_{N 1111}^{(d)}\right|_{\alpha=0}=\left.\frac{d}{2} \mathcal{D}^{+} I_{N 1122}^{(d)}\right|_{\alpha=0}, \\
\left.\partial_{-2 \alpha}^{2} I_{N 1111}^{(d)}\right|_{\alpha=0} & =\left.\frac{d}{4}\left(\partial_{-m_{1}^{2}}+\partial_{-m_{4}^{2}}+\partial_{-m_{5}^{2}}\right) \mathcal{D}^{+} I_{N 1111}^{(d)}\right|_{\alpha=0}+\left.\frac{d(d+2)}{4} \partial_{-m_{4}^{2}}^{2} \partial_{-m_{5}^{2}}^{2} \mathcal{D}^{++} I_{N 1111}^{(d)}\right|_{\alpha=0} \\
& =\left.\frac{d}{4} \mathcal{D}^{+}\left(N I_{N+1,1111}^{(d)}+I_{N 1121}^{(d)}+I_{N 1112}^{(d)}\right)\right|_{\alpha=0}+\left.d(d+2) \mathcal{D}^{++} I_{N 1133}^{(d)}\right|_{\alpha=0},
\end{aligned}
$$

where $\mathcal{D}^{+} I^{(d)} \equiv(4 \pi)^{3} I^{(d+2)}$ etc., such that Eqs. (2.10), (2.11) finally read

$$
\begin{aligned}
& \mathcal{F}_{P} \frac{1}{\left[P^{2}\right]^{N}} \Pi_{11}^{0 i} \Pi_{11}^{0 i}=\frac{d}{2} \mathcal{D}^{+} \sum_{P} \frac{\Pi_{12}^{0} \Pi_{12}^{0}}{\left[P^{2}\right]^{N}}, \\
& \mathcal{F}_{P} \frac{1}{\left[P^{2}\right]^{N}} \Pi_{11}^{i j} \Pi_{11}^{i j}=\frac{N d}{4} \mathcal{D}^{+} \sum_{P} \frac{\Pi_{11} \Pi_{11}}{\left[P^{2}\right]^{N+1}}+\frac{d}{2} \mathcal{D}^{+} \sum_{P} \frac{\Pi_{21} \Pi_{11}}{\left[P^{2}\right]^{N}}+d(d+2) \mathcal{D}^{++} \mathcal{F}_{P} \frac{\Pi_{31} \Pi_{31}}{\left[P^{2}\right]^{N}},
\end{aligned}
$$

and we obtain our final representation of Eq. (2.7) as

$$
\begin{aligned}
\mathcal{M}_{N,-2} & =4 \mathcal{f}_{P} \frac{\Pi_{11}^{00} \Pi_{11}^{00}}{P^{2 N}}+4 d \mathcal{D}^{+} \sum_{P} \frac{\Pi_{12}^{0} \Pi_{12}^{0}}{P^{2 N}}+N d \mathcal{D}^{+} \oint_{P} \frac{\Pi_{11} \Pi_{11}}{P^{2 N+2}}+2 d \mathcal{D}^{+} \oint_{P} \frac{\Pi_{21} \Pi_{11}}{P^{2 N}}+ \\
& +4 d(d+2) \mathcal{D}^{++} \sum_{P} \frac{\Pi_{31} \Pi_{31}}{P^{2 N}}-2 I_{1}^{0} \oint_{P} \frac{\Pi_{11}}{P^{2 N-2}}+2 I_{N}^{0} I_{1}^{0} I_{1}^{0} \\
& \equiv 4 V_{3}+4 d V_{4}+N d V_{5}+2 d V_{6}+4 d(d+2) V_{7}-2 I_{1}^{0} \oint_{P} \frac{\Pi_{11}}{P^{2 N-2}}+2 I_{N}^{0} I_{1}^{0} I_{1}^{0} .
\end{aligned}
$$

It remains to calculate $1 / 3 / 1$-loop spectacles-type sum-integrals $\left\{V_{3}, V_{4}, V_{5}, V_{6}, V_{7}\right\}$ in $d / d+$ $2 / d+4$ dimensions. Note however that their structure is quite similar, such that we will be able to employ a quite generic strategy for their evaluation, as will be explained below.

\subsection{Decomposition of spectacles-type sum-integrals}

Any spectacles-type sum-integral with two generic 2-point functions $\Pi_{1}(P), \Pi_{2}(P)$ can be identically rewritten as (suppressing indices of $\Pi$; for a full definition, see Eq. (3.13))

$$
\begin{aligned}
V & \equiv \oiint_{P} \frac{\left(P_{0}\right)^{m}}{\left[P^{2}\right]^{n}} \Pi_{1} \Pi_{2}=V^{\mathrm{f}(\text { inite })}+V^{\mathrm{d}(\text { ivergent })}+V^{\mathrm{z}(\text { ero })}, \\
V^{\mathrm{f}} & =\oint_{P}^{\prime} \frac{\left(P_{0}\right)^{m}}{\left[P^{2}\right]^{n}}\left\{\frac{1}{2}\left(\Pi_{1}-\Pi_{1}^{B}\right)\left(\Pi_{2}-\Pi_{2}^{B}\right)+\left(\Pi_{1}-\Pi_{1}^{C}\right)\left(\Pi_{2}^{B}-\Pi_{2}^{D}\right)\right\}+\{1 \leftrightarrow 2\}, \\
V^{\mathrm{d}} & =\mathcal{f}_{P}^{\prime} \frac{\left(P_{0}\right)^{m}}{\left[P^{2}\right]^{n}}\left\{\Pi_{1} \Pi_{2}^{D}-\Pi_{1}^{B} \Pi_{2}^{D}+\left(\Pi_{1}^{C}-\Pi_{1}^{B}\right)\left(\Pi_{2}^{B}-\Pi_{2}^{D}\right)+\frac{1}{2} \Pi_{1}^{B} \Pi_{2}^{B}\right\}+\{1 \leftrightarrow 2\}, \\
V^{\mathrm{z}} & =\sum_{P} \frac{\delta_{P_{0}}\left(P_{0}\right)^{m}}{\left[P^{2}\right]^{n}} \Pi_{1} \Pi_{2}=\delta_{m} \sum_{P} \frac{\delta_{P_{0}}}{\left[P^{2}\right]^{n}} \Pi_{1} \Pi_{2},
\end{aligned}
$$

where the $\Pi^{B}$ (T =0 part; leading UV div), $\Pi^{D}$ (integer power of $P^{2}$ such that $\left(\Pi^{B}-\Pi^{D}\right)$ is finite as $\epsilon \rightarrow 0$ ) and $\Pi^{C}$ (sub-leading UV divergence and sometimes further subtractions) are chosen such that the terms in $V^{\mathrm{f}}$ are finite (and could be evaluated numerically in coordinate space at $\epsilon=0$ ); those in $V^{\mathrm{d}}$ are simple 2-loop (first term) and trivial 1-loop structures; while $V^{\mathrm{z}}$ is the zero mode treated in Sec. 4. The subtraction terms $\Pi^{B, C, D}$ are detailed in Sec. 3.1. 


\section{Non-zero modes}

The goal of this section is to compute the $V^{\mathrm{d}}$ of Eq. (2.17) analytically, and to evaluate the $V^{\mathrm{f}}$ of Eq. (2.16) numerically, from simple low-dimensional integral representations.

\subsection{Generic formulae for 2-point subtraction terms}

For generic indices, let us specify the subtraction terms as

$$
\begin{aligned}
& \Pi_{a b 0}^{B} \equiv \int_{Q} \frac{1}{\left[Q^{2}\right]^{a}\left[(P+Q)^{2}\right]^{b}}=\frac{g_{00}(a, b, d+1)}{\left(P^{2}\right)^{a+b-(d+1) / 2}} \quad \text { with } \quad g_{00}(a, b, d)=G(a, b, d) \\
& \Pi_{a b 1}^{B} \equiv U_{\mu} \int_{Q} \frac{Q_{\mu}}{\left[Q^{2}\right]^{a}\left[(P+Q)^{2}\right]^{b}}=U_{\mu}\left\{P_{\mu} A\left(P^{2}\right)\right\}=P_{0} A\left(P^{2}\right) \\
&=\frac{P_{0} g_{10}(a, b, d+1)}{\left(P^{2}\right)^{a+b-(d+1) / 2}} \\
& \text { with } \quad g_{10}(a, b, d)=\frac{1}{2}[G(a, b-1, d)-G(a-1, b, d)-G(a, b, d)] \\
& \Pi_{a b 2}^{B} \equiv U_{\mu} U_{\nu} \int_{Q} \frac{Q_{\mu} Q_{\nu}}{\left[Q^{2}\right]^{a}\left[(P+Q)^{2}\right]^{b}}=U_{\mu} U_{\nu}\left\{g_{\mu \nu} A\left(P^{2}\right)+P_{\mu} P_{\nu} B\left(P^{2}\right)\right\}=A\left(P^{2}\right)+P_{0}^{2} B\left(P^{2}\right) \\
&=\frac{g_{21}(a, b, d+1)}{\left(P^{2}\right)^{a+b-(d+3) / 2}}+\frac{P_{0}^{2} g_{20}(a, b, d+1)}{\left(P^{2}\right)^{a+b-(d+1) / 2}} \\
& \text { with } \quad g_{21}(a, b, d)=[2 G(a-1, b, d)+2 G(a, b-1, d)+2 G(a-1, b-1, d)- \\
&\quad-G(a, b, d)-G(a-2, b, d)-G(a, b-2, d)] \frac{1}{4(d-1)}
\end{aligned}
$$

where the coefficient functions $g_{i j}$ derive from $4 \mathrm{~d}$ rotational invariance. Since efficient computation needs compact notation, we summarize the various $\Pi^{B}$ and $\Pi^{C}$ as given above as

$$
\begin{aligned}
\Pi_{a b c}^{B} & =\sum_{n=0}^{[c / 2]} \frac{\left(P_{0}\right)^{c-2 n} g_{c, n}(a, b, d+1)}{\left(P^{2}\right)^{a+b-(d+1) / 2-n}} \\
\Pi_{a b c}^{C}-\Pi_{a b c}^{B} & =\frac{1+(-1)^{c}}{2} \frac{I_{a}^{c}(d)}{\left(P^{2}\right)^{b}}+(-1)^{c} \sum_{n=0}^{[c / 2]}\left(\begin{array}{c}
c \\
2 n
\end{array}\right) I_{b}^{2 n}(d) \frac{\left(P_{0}\right)^{c-2 n}}{\left(P^{2}\right)^{a}},
\end{aligned}
$$

where $[c / 2]=\{c / 2,(c-1) / 2\}$ for $c=\{$ even, odd $\}$. 


\subsection{Generic formulae for divergences $V^{\mathrm{d}}$ of non-zero modes}

Due to the structure of $\Pi_{a b c}^{D}$, cf. Eq. (3.9), Eq. (2.17) factorizes as

$$
\begin{aligned}
V^{\mathrm{d}} & =\oint_{P}^{\prime} \frac{\left(P_{0}\right)^{m}}{\left[P^{2}\right]^{n}}\left\{\Pi_{1} \frac{\left(P^{2}\right)^{\epsilon}}{\left(\alpha_{2} T^{2}\right)^{\epsilon}}+\left(\Pi_{1}^{C}-\Pi_{1}^{B}\right)\left(1-\frac{\left(P^{2}\right)^{\epsilon}}{\left(\alpha_{2} T^{2}\right)^{\epsilon}}\right)+\frac{\Pi_{1}^{B}}{2}\left(1-\frac{2\left(P^{2}\right)^{\epsilon}}{\left(\alpha_{2} T^{2}\right)^{\epsilon}}\right)\right\} \Pi_{2}^{B}+ \\
& +\{1 \leftrightarrow 2\}
\end{aligned}
$$

where the $\alpha_{i}$ (cf. Eq. (3.9)) are constants that might be chosen such as to simplify the finite parts $V^{f}$, and which also serve to facilitate comparison with other approaches (e.g. $[11,12,20]$ ).

For the generic spectacles-type 3-loop sum-integral

$$
\begin{aligned}
V\left(s_{1 \ldots 5} ; s_{6} \ldots 8\right) & \equiv \oint_{P Q R} \frac{\left(P_{0}\right)^{s_{6}}\left(Q_{0}\right)^{s_{7}}\left(R_{0}\right)^{s_{8}}}{\left[P^{2}\right]^{s_{1}}\left[Q^{2}\right]^{s_{2}}\left[R^{2}\right]^{s_{3}}\left[(P+Q)^{2}\right]^{s_{4}}\left[(P+R)^{2}\right]^{s_{5}}} \\
& =\sum_{P} \frac{\left(P_{0}\right)^{s_{6}}}{\left[P^{2}\right]^{s_{1}}} \Pi_{s_{2} s_{4} s_{7}}(P) \Pi_{s_{3} s_{5} s_{8}}(P)
\end{aligned}
$$

we therefore write a general expression for the divergent parts of their non-zero modes:

$$
\begin{aligned}
V^{\mathrm{d}}\left(s_{1 \ldots 8}\right) & =\sum_{n=0}^{\left[s_{8} / 2\right]} g_{s_{8}, n}\left(s_{3}, s_{5}, d+1\right) f_{\alpha_{2}}\left(s_{135}-\frac{d+1}{2}-n, s_{2}, s_{4}, s_{68}-2 n, s_{7} ; d\right)+ \\
& +\sum_{n=0}^{\left[s_{7} / 2\right]} g_{s_{7}, n}\left(s_{2}, s_{4}, d+1\right) f_{\alpha_{1}}\left(s_{124}-\frac{d+1}{2}-n, s_{3}, s_{5}, s_{67}-2 n, s_{8} ; d\right)
\end{aligned}
$$

( $\alpha_{i}$-independence follows when adding $V_{I I}^{\mathrm{f}}$, cf. Eq. (D.35)) where the functions $f$ are given by

$$
\begin{aligned}
f_{\alpha}\left(s_{1 \ldots 5} ; d\right) \equiv & \mathcal{f}_{P}^{\prime} \frac{\left(P_{0}\right)^{s_{4}}}{\left(P^{2}\right)^{s_{1}}}\{\text { see Eq. }(3.12)\}_{s_{235}} \\
= & \frac{1}{\left(\alpha T^{2}\right)^{\epsilon}} L^{\prime}\left(s_{1}-\epsilon, s_{2 \ldots 5}, d\right)+I_{s_{2}}^{s_{5}}(d) \hat{I}_{s_{13}}^{s_{4}}(d, \alpha)+ \\
+ & \sum_{n=0}^{\left[s_{5} / 2\right]}\left[(-1)^{s_{5}}\left(\begin{array}{c}
s_{5} \\
2 n
\end{array}\right) I_{s_{3}}^{2 n}(d) \hat{I}_{s_{12}}^{s_{45}-2 n}(d, \alpha)+\right. \\
& \left.\quad+\frac{1}{2} g_{s_{5}, n}\left(s_{2}, s_{3}, d+1\right) \tilde{I}_{s_{123}-(d+1) / 2-n}^{s_{4}+s_{5}-2 n}(d, \alpha)\right]
\end{aligned}
$$

with the one- and two-loop structures

$$
\begin{aligned}
\hat{I}_{a}^{b}(d, \alpha) & \equiv I_{a}^{b}(d)-\left(\alpha T^{2}\right)^{-\epsilon} I_{a-\epsilon}^{b}(d), \\
\tilde{I}_{a}^{b}(d, \alpha) & \equiv I_{a}^{b}(d)-2\left(\alpha T^{2}\right)^{-\epsilon} I_{a-\epsilon}^{b}(d), \\
L^{\prime}\left(s_{1 \ldots 5}, d\right) & \equiv L\left(s_{1 \ldots 5}, d\right)-\delta_{s_{4}} A\left(s_{1 \ldots 3}, s_{5}, d\right) .
\end{aligned}
$$

The latter reduces to 1-loop sum-integrals as $L \rightarrow I \cdot I$, as can be derived systematically via IBP; the cases relevant for the present computation are given in appendix B. 


\subsection{Specific results for divergences $V^{\mathrm{d}}$ of non-zero modes}

From Eq. (3.14), we obtain the desired expansions for the divergent pieces of $\left\{V_{3}, V_{4}, V_{5}, V_{6}, V_{7}\right\}$ needed for the decomposition Eq. (2.14) of the specific sum-integral $\mathcal{M}_{3,-2}$ around $d=3-2 \epsilon$,

$$
\begin{array}{ll}
V_{3}^{\mathrm{d}}=V^{\mathrm{d}}(31111 ; 022) & \approx \frac{T^{2}}{(4 \pi)^{4}} \frac{\left(4 \pi T^{2}\right)^{-3 \epsilon}}{\epsilon^{2}} \frac{1}{288}\left[1+\left(\frac{73}{12}+\gamma_{\mathrm{E}}+24 \ln G\right) \epsilon+\mathcal{O}\left(\epsilon^{2}\right)\right], \\
V_{4}^{\mathrm{d}}=\mathcal{D}^{+} V^{\mathrm{d}}(31122 ; 011) & \approx \frac{T^{2}}{(4 \pi)^{4}} \frac{\left(4 \pi T^{2}\right)^{-3 \epsilon}}{\epsilon^{2}}\left[0-\frac{1}{162} \epsilon+\mathcal{O}\left(\epsilon^{2}\right)\right], \\
V_{5}^{\mathrm{d}}=\mathcal{D}^{+} V^{\mathrm{d}}(41111) & \approx \frac{T^{2}}{(4 \pi)^{4}} \frac{\left(4 \pi T^{2}\right)^{-3 \epsilon}}{\epsilon^{2}} \frac{1}{432}\left[1+\left(\frac{34}{3}-11 \gamma_{\mathrm{E}}-120 \ln G+24 \ln (2 \pi)\right) \epsilon\right], \\
V_{6}^{\mathrm{d}}=\mathcal{D}^{+} V^{\mathrm{d}}(32111) & \approx \frac{T^{2}}{(4 \pi)^{4}} \frac{\left(4 \pi T^{2}\right)^{-3 \epsilon}}{\epsilon^{2}} \frac{-1}{96}\left[1+\left(\frac{139}{18}-\frac{17}{3} \gamma_{\mathrm{E}}-56 \ln G+\frac{40}{3} \ln (2 \pi)\right) \epsilon\right], \\
V_{7}^{\mathrm{d}}=\mathcal{D}^{++} V^{\mathrm{d}}(33311) & \approx \frac{T^{2}}{(4 \pi)^{4}} \frac{\left(4 \pi T^{2}\right)^{-3 \epsilon}}{\epsilon^{2}} \frac{1}{432}\left[1+\left(\frac{26}{5}-\frac{13}{5} \gamma_{\mathrm{E}}-\frac{96}{5} \ln G+\frac{36}{5} \ln (2 \pi)\right) \epsilon\right],
\end{array}
$$

where $V^{\mathrm{d}}(41111) \equiv V^{\mathrm{d}}(41111,000)$ etc., the Glaisher constant $G$ appears inside a logarithm, with $12 \ln (G)=1+\zeta^{\prime}(-1) / \zeta(-1)$, and we refrain from listing the somewhat lengthy constant pieces, which contain the dependence on the arbitrary constants $\alpha_{i}$ as defined in Eq. (3.12) as well. As mentioned above, this dependence will precisely cancel that in $V^{\mathrm{f}}$, which is contained in $V_{I I}^{\mathrm{f}}$, cf. Eq. (D.35).

\subsection{Finite parts $V^{\mathrm{f}}$ of non-zero modes}

With the structure of the subtracted terms as in Eq. (2.16), they contribute to $\mathcal{M}_{3,-2}$ as

$$
\begin{aligned}
\mathcal{M}_{3,-2}^{\mathrm{nz}, \mathrm{f}} & =4 V_{3}^{\mathrm{f}}(31111,022)+12 \mathcal{D}^{+} V_{4}^{\mathrm{f}}(31122,011)+9 \mathcal{D}^{+} V_{5}^{\mathrm{f}}(41111)+ \\
& +6 \mathcal{D}^{+} V_{6}^{\mathrm{f}}(32111)+60 \mathcal{D}^{++} V_{7}^{\mathrm{f}}(33311)+\mathcal{O}(\epsilon) \\
& \approx \frac{T^{2}}{(4 \pi)^{4}}\left[n_{1}+\mathcal{O}(\epsilon)\right], \quad n_{1} \approx+0.0645513(1)
\end{aligned}
$$

where we discuss the numerical evaluation of $n_{1}$, for which coordinate-space methods prove useful, in App. D.1. The value given above was obtained setting all $\alpha_{i}=16 \pi^{2} e^{3 / 2-\gamma}$.

\section{Zero modes}

Here, we treat the $P_{0}=0$ modes of all 3-loop sum-integrals of Eq. (2.14) that are needed for the specific case $\mathcal{M}_{3,-2}$, while discussing the generic strategy. In terms of

$$
S\left(s_{1 \ldots 5} ; s_{6}, s_{7}\right) \equiv \sum_{P} \frac{\delta_{P_{0}}}{\left[P^{2}\right]^{s_{1}}} \Pi_{s_{2} s_{4} s_{6}} \Pi_{s_{3} s_{5} s_{7}} \quad, \quad \Pi_{a b c} \equiv \mathcal{F}_{Q} \frac{Q_{0}^{c}}{\left[Q^{2}\right]^{a}\left[(P+Q)^{2}\right]^{b}},
$$

and using the IBP relations of App. C to improve their IR behavior, they read

$$
V_{3}^{\mathrm{Z}}=S(31111 ; 22) \quad=\frac{2}{d-8}\left[\frac{S(12121 ; 22)+S(12211 ; 22)-I_{2}^{2} A(221 ; 2, d)}{(d-5)}-I_{2}^{2} A(311 ; 2, d)\right],
$$




$$
\begin{aligned}
& V_{4}^{\mathrm{z}}=\mathcal{D}^{+} S(31122 ; 11)=0 \text { after summation in } \delta_{P_{0}} \Pi_{a b 1}, \\
& V_{5}^{\mathrm{z}}=\mathcal{D}^{+} S(41111)=\frac{2}{d-8}\left[V_{6}^{z}-\mathcal{D}^{+}\left(I_{2}^{0} A(411 ; 0, d)\right)\right], \\
& V_{6}^{\mathrm{z}}=\mathcal{D}^{+} S(32111) \quad=\frac{1}{d-5}\left[\mathcal{D}^{+} S(22121)+\frac{2}{d-2} \mathcal{D}^{+} S(12221)-\mathcal{D}^{+}\left(I_{2}^{0} A(321 ; 0, d)\right)\right], \\
& V_{7}^{\mathrm{z}}=\mathcal{D}^{++} S(33311)=2 \frac{\left(d^{2}-13 d+38\right) \mathcal{D}^{++} S(23321)-(d-10) \mathcal{D}^{++} S(23222)}{(d-2)(d-5)(d-8)} .
\end{aligned}
$$

A generic decomposition into finite part (first line) and remainder of zero-mode integrals $S$ is

$$
\begin{aligned}
S\left(s_{1 \ldots 5} ; s_{6}, s_{7}\right) & =\sum_{P} \frac{\delta_{P_{0}}}{\left[P^{2}\right]^{s_{1}}}\left[\Pi_{s_{2} s_{4} s_{6}}-\Pi_{s_{2} s_{4} s_{6}}^{A}\right]\left[\Pi_{s_{3} s_{5} s_{7}}-\Pi_{s_{3} s_{5} s_{7}}^{A}\right]+ \\
& +\sum_{P} \frac{\delta_{P_{0}}}{\left[P^{2}\right]^{s_{1}}} \Pi_{s_{2} s_{4} s_{6}} \Pi_{s_{3} s_{5} s_{7}}^{A}+\sum_{P} \frac{\delta_{P_{0}}}{\left[P^{2}\right]^{s_{1}}} \Pi_{s_{2} s_{4} s_{6}}^{A} \Pi_{s_{3} s_{5} s_{7}}- \\
& -\sum_{P} \frac{\delta_{P_{0}}}{\left[P^{2}\right]^{s_{1}}} \Pi_{s_{2} s_{4} s_{6}}^{A} \Pi_{s_{3} s_{5} s_{7}}^{A} \equiv S^{\mathrm{f}(\text { inite })}+S^{\mathrm{d} \text { (ivergent) }},
\end{aligned}
$$

where the subtraction terms are defined as

$$
\begin{aligned}
\Pi_{a b c}^{A} & \equiv \theta(d+2-2 a-2 b+c) \delta_{P_{0}} \Pi_{a b c}^{B}+\theta(2 a+2 b-c-2-d) \delta_{P_{0}} \oint_{Q} \frac{\delta_{Q_{0}} Q_{0}^{c}}{\left[Q^{2}\right]^{a}\left[(P-Q)^{2}\right]^{b}} \\
& =\theta(d+2-2 a-2 b+c) \delta_{P_{0}} \Pi_{a b c}^{B}+\theta(2 a+2 b-c-2-d) \delta_{P_{0}} \delta_{c} \frac{T G(a, b, d)}{\left(P^{2}\right)^{a+b-d / 2}},
\end{aligned}
$$

which represents subtraction of the usual leading UV divergence $\Pi^{B}$ given by the $T=0$ piece, whose analytical representation was given in Eq. (3.10), as well as subtraction of the zero-mode inside two-point functions $\Pi_{a b 0}$.

\subsection{Generic results for divergences $S^{\mathrm{d}}$ of zero modes}

Noting that the subtraction terms $\Pi_{a b c}^{A}$ are proportional to powers of $\mathbf{p}^{2}$, the last term of Eq. (4.7) vanishes identically since it is scale-free, while the other two can be expressed analytically using the 2-loop function $A$ :

$$
\begin{aligned}
S^{\mathrm{d}}\left(s_{1 \ldots 5} ; s_{6}, s_{7}, d\right) & =a\left(s_{1 \ldots 7}, d\right)+a\left(s_{1325476}, d\right)+0_{\text {scalefree }} \\
a\left(s_{1 \ldots 7}, d\right) & =\theta\left(d+2-2 s_{24}+s_{6}\right) e_{s_{6}} g_{s_{6}, s_{6} / 2}\left(s_{2}, s_{4}, d+1\right) A\left(s_{124}-\frac{d+1+s_{6}}{2}, s_{3}, s_{5}, s_{7}, d\right)+ \\
& +\theta\left(2 s_{24}-s_{6}-2-d\right) \delta_{s_{6}} T G\left(s_{2}, s_{4}, d\right) A\left(s_{124}-\frac{d}{2}, s_{3}, s_{5}, s_{7}, d\right),
\end{aligned}
$$

where $e_{s} \equiv\left(1+(-1)^{s}\right) / 2$ is $1(0)$ for even(odd) index.

\subsection{Specific results for divergences $V^{\mathrm{z}, \mathrm{d}}$ of zero modes}

Collecting and expanding around $d=3-2 \epsilon$ dimensions, we finally get for the divergent pieces of the zero modes

$$
V_{3}^{\mathrm{z}, \mathrm{d}} \approx \frac{T^{2}}{(4 \pi)^{4}} \frac{\left(4 \pi T^{2}\right)^{-3 \epsilon}}{\epsilon^{2}} \frac{-1}{48}\left[0+1 \epsilon+\left(\frac{49}{15}+\frac{\pi^{2}}{20}-3 \gamma_{\mathrm{E}}+6 \ln (2 \pi)\right) \epsilon^{2}+\mathcal{O}\left(\epsilon^{3}\right)\right]
$$




$$
\begin{aligned}
& V_{4}^{\mathrm{Z}, \mathrm{d}}=0, \\
& V_{5}^{\mathrm{Z}, \mathrm{d}} \approx \frac{T^{2}}{(4 \pi)^{4}} \frac{\left(4 \pi T^{2}\right)^{-3 \epsilon}}{\epsilon^{2}} \frac{-1}{72}\left[1+\left(\frac{5}{3}-\gamma_{\mathrm{E}}+4 \ln (2 \pi)\right) \epsilon+\mathcal{O}\left(\epsilon^{2}\right)\right], \\
& V_{6}^{\mathrm{Z}, \mathrm{d}} \approx \frac{T^{2}}{(4 \pi)^{4}} \frac{\left(4 \pi T^{2}\right)^{-3 \epsilon}}{\epsilon^{2}} \frac{5}{144}\left[1+\left(\frac{31}{15}-\gamma_{\mathrm{E}}+4 \ln (2 \pi)\right) \epsilon+\mathcal{O}\left(\epsilon^{2}\right)\right], \\
& V_{7}^{\mathrm{Z}, \mathrm{d}} \approx \frac{T^{2}}{(4 \pi)^{4}} \frac{\left(4 \pi T^{2}\right)^{-3 \epsilon}}{\epsilon^{2}} \frac{-1}{240}\left[1+\left(\frac{41}{15}-\gamma_{\mathrm{E}}+4 \ln (2 \pi)\right) \epsilon+\mathcal{O}\left(\epsilon^{2}\right)\right] .
\end{aligned}
$$

Here, we have of course assumed that all six subtracted sum-integrals shown in the first line of Eq. (4.7) are finite. We prove this assertion by explicit computation in App. D.2, with results given in Eq. (4.16) below.

\subsection{Finite parts $V^{z, f}$ of zero modes}

We now have to write down integral representations for the $\Pi^{A}$-subtracted parts of our zeromodes and solve them numerically, to prove that they are finite indeed. The first line of Eq. (4.7), to be evaluated numerically at $d=3$, contributes to $\mathcal{M}_{3,-2}$ as

$$
\begin{aligned}
\mathcal{M}_{3,-2}^{\mathrm{z,f}} & =\frac{4}{5} \sum_{P} \frac{\delta_{P_{0}}}{P^{2}}\left\{\left[\Pi_{222}-\Pi_{222}^{B}\right]\left[\Pi_{112}-\Pi_{112}^{B}\right]+\left[\Pi_{212}-\Pi_{212}^{B}\right]^{2}\right\}- \\
& -\frac{6}{5} \mathcal{D}^{+} \mathcal{\psi}_{P} \frac{\delta_{P_{0}}}{\left[P^{2}\right]^{2}}\left\{\left[\Pi_{220}-\Pi_{220}^{A}\right]\left[\Pi_{110}-\Pi_{110}^{A}\right]+2 P^{2}\left[\Pi_{220}-\Pi_{220}^{A}\right]\left[\Pi_{210}-\Pi_{210}^{A}\right]\right\}+ \\
& +\mathcal{D}^{++} \oint_{P} \frac{\delta_{P_{0}}}{\left[P^{2}\right]^{2}}\left\{96\left[\Pi_{320}-\Pi_{320}^{A}\right]\left[\Pi_{310}-\Pi_{310}^{A}\right]+84\left[\Pi_{320}-\Pi_{320}^{A}\right]\left[\Pi_{220}-\Pi_{220}^{A}\right]\right\} \\
& \approx \frac{T^{2}}{(4 \pi)^{4}}\left[n_{2}+\mathcal{O}(\epsilon)\right], \quad n_{2} \approx+0.24983747686(1),
\end{aligned}
$$

where we discuss the necessary integral representations as well as their numerical evaluation leading to $n_{2}$ in App. D.2.

\section{Result for $\mathcal{M}_{3,-2}$}

According to Eqs. (2.15)-(2.18) and Eqs. (4.2)-(4.7), each scalar spectacle-type sum-integral is decomposed as $V=V^{\mathrm{f}}+V^{\mathrm{d}}+V^{\mathrm{z}, \mathrm{f}}+V^{\mathrm{z}, \mathrm{d}}$, and according to Eq. (2.14) (with Eq. (B.6) for the 2-loop case therein), the master integral $\mathcal{M}_{3,-2}$ can be written exclusively in terms of such scalar spectacle-types and trivial 1-loop integrals. Collecting and expanding, we finally obtain

$$
\begin{aligned}
\mu^{6 \epsilon} \mathcal{M}_{3,-2} & \approx \frac{T^{2}}{(4 \pi)^{4}}\left(\frac{\mu^{2}}{4 \pi T^{2}}\right)^{3 \epsilon} \frac{1}{\epsilon^{2}}\left[-\frac{5}{36}-\left(\frac{11}{216}+\frac{5}{36} \gamma_{\mathrm{E}}+\frac{10}{3} \ln G\right) \epsilon+m \epsilon^{2}+\mathcal{O}\left(\epsilon^{3}\right)\right], \quad(5.1) \\
\text { with } m & =\frac{59}{405}+\frac{83}{72}{\gamma_{\mathrm{E}}}^{2}+\gamma_{E}\left(-\frac{559}{1080}+\frac{2}{3} \ln (2 \pi)-\frac{2}{3} \ln G+\frac{7}{1080} \zeta(3)-\frac{1}{3780} \zeta(5)\right)- \\
& -\frac{757}{2160} \pi^{2}-\frac{2}{3} \ln ^{2} 2-\frac{65}{9} \ln G+\frac{7}{15} \ln (2 \pi)-\frac{2}{3} \ln (4 \pi) \ln \pi+\frac{28}{9} \gamma_{1}+
\end{aligned}
$$




$$
\begin{aligned}
& +\frac{37}{3240} \zeta(3)+\frac{113}{226800} \zeta(5)-\frac{7}{540} \zeta^{\prime}(3)+\frac{1}{1890} \zeta^{\prime}(5)+2 \zeta^{\prime \prime}(-1)+n_{1}+n_{2} \\
& \approx n_{1}+n_{2}-6.5129800346005775127 \approx-6.1985913(1),
\end{aligned}
$$

where the Glaisher constant $G$ appears inside a logarithm, with $12 \ln (G)=1+\zeta^{\prime}(-1) / \zeta(-1)$, and various zeta values as well as the Stieltjes constant $\gamma_{1}$, defined by $\zeta(1+\epsilon)=1 / \epsilon+\gamma_{\mathrm{E}}-$ $\gamma_{1} \epsilon+\mathcal{O}\left(\epsilon^{2}\right)$, enter the constant term.

While it might at first sight seem that we have used an unnecessarily generic notation in our decomposition and treatment of the various contributing terms, let us remark that this was done in order to provide a setup that allows for solving many more sum-integrals than just the special case $\mathcal{M}_{3,-2}$ given above. Indeed, as we quickly demonstrate in App. E, the two previously known sum-integrals $\mathcal{M}_{1,0}$ [17] and $V_{2}$ [12] (which each had required quite some effort to evaluate) are reproduced by our generic formulae, and there is no doubt that the same could be said about $\mathcal{M}_{2,-2}[2,3,11]$, although we have not explicitly checked that.

\section{Conclusions}

In this work, we have made important progress in two respects. First, by transferring proven technology from zero-temperature field theory to the finite-temperature case (the $T$-operators of Sec. 2.2), we were able to map tensor sum-integrals onto scalar ones. Naturally, although we have tested this method on a specific case only, it is applicable much more generally. Much in the spirit of Ref. [22], by using the new tensor technique we have dissected the problem to its clean core, finding a class of spectacles-type sum-integrals that are amenable to known techniques.

Second, we have worked out generic solutions for these massless bosonic 3-loop spectaclestype sum-integrals, which are applicable to a wide class of such integrals. Let us note that this is only the third instance of treating a more generic class of sum-integrals in a single computation (the first two such instance were recorded in $[8,9]$ ), and represents the first steps beyond the case-by-case analyses found in the literature - a development urgently needed in order to close the glaring gap between well-established generic integration techniques in zerotemperature field theory and the few painstakingly derived cases that are known at finite temperatures.

A generalization of our results to sum-integrals involving fermions should be possible (at least for vanishing masses and zero chemical potentials) in a straightforward manner, and could in fact even turn out to be structurally simpler due to the absence of zero-modes in fermionic lines, which had cost us quite some effort in the bosonic case as treated in Sec. 4 above. There are a number of well-defined applications that await the evaluation of such fermionic sum-integrals, such as the $N_{\mathrm{f}} \neq 0$ parts of EQCD matching coefficients as listed in [9].

As an application, of our generic formulae, we have given results for the new massless 3-loop sum-integral $\mathcal{M}_{3,-2}$, which represents a key building block of the bosonic part of the Debye screening mass in hot QCD $[9,15]$. Reassuringly, our generic formalism reproduces 
the two previously known sum-integrals of the spectacles-type class without effort, as we have demonstrated in App. E.

\section{Acknowledgments}

Y.S. thanks Hide and Shoko Goyahso for hospitality while part of this work was done. The work of I.G. is supported by the Deutsche Forschungsgemeinschaft (DFG) under grant no. GRK 881. Y.S. is supported by the Heisenberg program of the DFG, under contract no. SCHR 993/1.

\section{A Standard integrals}

For convenience, we collect here some of the basic functions used above, as defined in [8]. They are the massless 1-loop propagator at zero temperature

$$
G\left(s_{1}, s_{2}, d\right) \equiv\left(p^{2}\right)^{s_{12}-\frac{d}{2}} \int \frac{\mathrm{d}^{d} q}{(2 \pi)^{d}} \frac{1}{\left[q^{2}\right]^{s_{1}}\left[(q-p)^{2}\right]^{s_{2}}}=\frac{\Gamma\left(\frac{d}{2}-s_{1}\right) \Gamma\left(\frac{d}{2}-s_{2}\right) \Gamma\left(s_{12}-\frac{d}{2}\right)}{(4 \pi)^{d / 2} \Gamma\left(s_{1}\right) \Gamma\left(s_{2}\right) \Gamma\left(d-s_{12}\right)} ;
$$

the 1-loop bosonic tadpoles

$$
I_{s}^{a} \equiv \sum_{Q} \frac{\left|Q_{0}\right|^{a}}{\left[Q^{2}\right]^{s}}=\frac{2 T \zeta(2 s-a-d)}{(2 \pi T)^{2 s-a-d}} \frac{\Gamma\left(s-\frac{d}{2}\right)}{(4 \pi)^{d / 2} \Gamma(s)}, \quad I_{s} \equiv \sum_{Q} \frac{1}{\left[Q^{2}\right]^{s}}=I_{s}^{0} ；
$$

a specific 2-loop tadpole

$$
\begin{aligned}
A\left(s_{1}, s_{2}, s_{3} ; s_{4}, d\right) & \equiv \oint_{P Q} \frac{\delta_{Q_{0}}\left|P_{0}\right|^{s_{4}}}{\left[Q^{2}\right]^{s_{1}}\left[P^{2}\right]^{s_{2}}\left[(P-Q)^{2}\right]^{s_{3}}} \\
& =\frac{2 T^{2} \zeta\left(2 s_{123}-2 d-s_{4}\right)}{(2 \pi T)^{2 s_{123}-2 d-s_{4}}} \frac{\Gamma\left(s_{13}-\frac{d}{2}\right) \Gamma\left(s_{12}-\frac{d}{2}\right) \Gamma\left(\frac{d}{2}-s_{1}\right) \Gamma\left(s_{123}-d\right)}{(4 \pi)^{d} \Gamma\left(s_{2}\right) \Gamma\left(s_{3}\right) \Gamma(d / 2) \Gamma\left(s_{1123}-d\right)},
\end{aligned}
$$

where the shorthand $s_{a b c \ldots} \equiv s_{c}+s_{b}+s_{c}+\ldots$.

\section{B IBP relations for 2-loop sum-integrals}

It is known that, for integer indices $s_{1 \ldots 5}$, all 2-loop sum-integrals of the form

$$
L\left(s_{1 \ldots .}, d\right) \equiv \sum_{P Q} \frac{\left(P_{0}\right)^{s_{4}}\left(Q_{0}\right)^{s_{5}}}{\left[P^{2}\right]^{s_{1}}\left[Q^{2}\right]^{s_{2}}\left[(P+Q)^{2}\right]^{s_{3}}}
$$

are trivial in the sense that by systematic use of IBP relations [10] they reduce to products

of 1-loop sum-integrals (which are in turn known analytically, cf. Eq. (A.2)). We read the specific bosonic cases that are needed for the present calculation from our algorithmically generated tables, to get

$$
L(331,00, d)=-\frac{12(d-8)(d-5)}{(d-2)(d-4)(d-9)(d-11)} I_{4}^{0}(d) I_{3}^{0}(d),
$$




$$
\begin{aligned}
& L(311,00, d)=-\frac{4}{(d-2)(d-7)} I_{3}^{0}(d) I_{2}^{0}(d) \\
& L(221,00, d)=0 \\
& L(312,11, d)=\frac{(d-6)(d-5)(d-3)}{2(d-9)(d-7)(d-2)} I_{3}^{0}(d) I_{2}^{0}(d) \\
& L(211,00, d)=-\frac{1}{(d-5)(d-2)} I_{2}^{0}(d) I_{2}^{0}(d) \\
& L(211,02, d)=\frac{d-3}{d-5} I_{2}^{0}(d) I_{1}^{0}(d) \\
& L(311,22, d)=-\frac{(d-4)\left(d^{2}-8 d+19\right)}{4(d-7)(d-5)} I_{2}^{0}(d) I_{1}^{0}(d) .
\end{aligned}
$$

\section{IBP relations for 3-loop zero modes}

For the zero-mode sum-integrals $S$ defined in Eq. (4.1), for which the generic IBP relation

$$
\begin{aligned}
0 & =\partial_{\mathbf{p}} \mathbf{p} \circ S\left(s_{1 \ldots 5} ; s_{6}, s_{7}\right) \\
& =\left\{\left(d-2 s_{1}-s_{4}-s_{5}\right)+s_{4} \mathbf{4}^{+}\left(\mathbf{2}^{-}-\mathbf{1}^{-}\right)+s_{5} \mathbf{5}^{+}\left(\mathbf{3}^{-}-\mathbf{1}^{-}\right)\right\} \circ S\left(s_{1 \ldots 5} ; s_{6}, s_{7}\right)
\end{aligned}
$$

holds and the additional symmetries $\delta_{P_{0}} \Pi_{a b, \text { odd }}=0$ and $\delta_{P_{0}} \Pi_{a b c}=(-1)^{c} \delta_{P_{0}} \Pi_{b a c}$ can be used, we give a number of linear relations here that prove useful in Sec. 4 of the main text. Acting with the IBP Eq. (C.1) on the sum $\frac{d-2 n+1}{2} S(n 1111 ; e e)+S(n-1,2111 ; e e)$ (with $e$ an even integer) and using symmetries gives the generic relation

$$
\begin{aligned}
0 & =\frac{1}{2}(d-2 n+1)(d-2 n-2) S(n 1111 ; e e)-S(n-2,2121 ; e e)-S(n-2,2211 ; e e)+ \\
& +(d-2 n+1) I_{2}^{e} A(n 11 ; e, d)+I_{2}^{e} A(n-1,21 ; e, d)
\end{aligned}
$$

which when applied at $\{n, e\}=\{3,2\}$ gives

$$
\begin{aligned}
0 & =\frac{1}{2}(d-5)(d-8) S(31111 ; 22)-S(12121 ; 22)-S(12211 ; 22)+ \\
& +(d-5) I_{2}^{2} A(311 ; 2, d)+I_{2}^{2} A(221 ; 2, d) .
\end{aligned}
$$

In complete analogy, acting with the IBP Eq. (C.1) on $S(41111)$, on the sum $S(32111)+$ $\frac{1}{d-4} S(22211)$, as well as on the sum $S(32222)-(d-10) S(33212)+\frac{d^{2}-19 d+86}{2} S(33311)$ and using symmetries gives

$$
\begin{aligned}
& 0=(d-10) S(41111)-2 S(32111)+2 I_{2}^{0} A(411 ; 0, d) \\
& 0=(d-7) S(32111)-S(22121)-\frac{2}{d-4} S(12221)+I_{2}^{0} A(321 ; 0, d) \\
& 0=\frac{1}{2}(d-6)(d-9)(d-12) S(33311)+(d-14) S(23222)-\left(d^{2}-21 d+106\right) S(23321) .
\end{aligned}
$$




\section{Numerical evaluation of finite integrals}

In this Appendix, we treat the finite parts of non-zero (D.1) and zero-modes (D.2) that are needed in the main text, cf. Eqs. (3.19) and (4.16).

\section{D.1 Contribution to $\mathcal{M}_{3,-2}$ from finite parts $V^{\mathrm{f}}$ of non-zero modes}

Here, we discuss the evaluation of the finite integrals $V^{\mathrm{f}}$ as defined in Eq. (2.16), and as needed for Eq. (3.19) of the main text. They can be treated at $\epsilon=0$ in coordinate space. Note, however, that we need some of them in shifted dimensions (in fact, for $d \in\{3,5,7\}$ ). We will first provide the (inverse, spatial) Fourier transforms of propagators $1 / P^{2}$ as well as two-point functions $\Pi(P)$, and then reduce the coordinate-space representation of the $V^{\mathrm{f}}$ to simple integrals as far as possible, once more in a generic way with unspecified indices. We will profit from the fact that we are interested in odd $d$, in which case the Bessel functions coming from the Fourier transforms reduce to Bessel polynomials. The specific cases that we need for $\mathcal{M}_{3,-2}$ are then approximated numerically.

The $d$-dimensional angular averages $(\operatorname{Re}(\mathrm{d})>1)$ result in Bessel functions of first kind

$$
\begin{aligned}
\int \mathrm{d}^{d} \mathbf{r} f(r) e^{i \mathbf{p r}} & =\int_{0}^{\infty} \mathrm{d} r r^{d-1} f(r) \frac{2 \pi^{\frac{d-1}{2}}}{\Gamma\left(\frac{d-1}{2}\right)} \int_{-1}^{1} \mathrm{~d} u\left(1-u^{2}\right)^{\frac{d-3}{2}} e^{i p r u} \\
& =\int_{0}^{\infty} \mathrm{d} r r^{d-1} f(r) 2(2 \pi)^{\frac{d-1}{2}}(p r)^{2-d} j_{\frac{d}{2}-1}(p r) \\
& \text { with } j_{n}(x)=\sqrt{\frac{\pi}{2}} x^{n} J_{n}(x), \\
& j_{\left\{\frac{1}{2}, \frac{3}{2}, \frac{5}{2}\right\}}=\left\{\sin (x), \sin (x)-x \cos (x), 3 \sin (x)-3 x \cos (x)-x^{2} \sin (x)\right\}
\end{aligned}
$$

such that propagators transform into modified Bessel functions of 2 nd kind $(0<\operatorname{Re}(d)<4 s+1)$

$$
\begin{aligned}
\frac{1}{\left(\mathbf{p}^{2}+m^{2}\right)^{s}} & =\int \mathrm{d}^{d} \mathbf{r} e^{i \mathbf{p r}} \int \frac{\mathrm{d}^{d} \mathbf{k}}{(2 \pi)^{d}} \frac{e^{-i \mathbf{k r}}}{\left(\mathbf{k}^{2}+m^{2}\right)^{s}}=\int \mathrm{d}^{d} \mathbf{r} e^{i \mathbf{p r}} \frac{2 r^{2 s-d}}{(2 \pi)^{\frac{d+1}{2}}} \int_{0}^{\infty} \mathrm{d} k \frac{k j_{\frac{d}{2}-1}(k)}{\left(k^{2}+m^{2} r^{2}\right)^{s}} \\
& =\int \mathrm{d}^{d} \mathbf{r} e^{i \mathbf{p r}} \frac{2^{1-s}}{(2 \pi)^{\frac{d}{2}}} \frac{1}{\Gamma(s)}\left(\frac{m^{2}}{r^{2}}\right)^{\frac{d-2 s}{4}} K_{s-\frac{d}{2}}\left(\sqrt{m^{2} r^{2}}\right) \\
& =\int \mathrm{d}^{d} \mathbf{r} e^{i \mathbf{p r}} \frac{2^{-s}}{(2 \pi)^{\frac{d-1}{2}}} \frac{1}{\Gamma(s)}\left(\frac{m^{2}}{r^{2}}\right)^{\frac{d-2 s}{4}} \frac{e^{-\sqrt{m^{2} r^{2}}}}{\left(m^{2} r^{2}\right)^{\frac{1}{4}}} \kappa_{s-\frac{d}{2}}\left(\sqrt{m^{2} r^{2}}\right) \\
& \text { with } \kappa_{n}(x)=\sqrt{\frac{2 x}{\pi}} e^{x} K_{n}(x), \\
& \kappa_{ \pm\left\{\frac{1}{2}, \frac{3}{2}, \frac{5}{2}\right\}}(x)=\left\{1,1+\frac{1}{x}, 1+\frac{3}{x}+\frac{3}{x^{2}}\right\}
\end{aligned}
$$


and we get the Fourier transforms ${ }^{1}$

$$
\begin{aligned}
& \Pi_{s_{123}}^{(d)}(P) \equiv T \sum_{Q_{0}} \int \frac{\mathrm{d}^{\mathrm{d}} \mathbf{q}}{(2 \pi)^{d}} \frac{\left(Q_{0}\right)^{s_{3}}}{\left[Q^{2}\right]^{s_{1}}\left[(P+Q)^{2}\right]^{s_{2}}} \\
&=\int \mathrm{d}^{d} \mathbf{r} e^{i \mathbf{p r}} e^{-\left|P_{0}\right| r} \frac{T^{d}(2 \pi T)^{d+1+s_{3}-2 s_{12}}}{2^{s_{12} \Gamma\left(s_{1}\right) \Gamma\left(s_{2}\right)(2 \pi T r)^{d+1-s_{12}}}} \hat{f}_{d, s_{123}}^{\Pi}\left(2 \pi T r, \frac{P_{0}}{2 \pi T}\right) \\
& \text { with } \hat{f}_{d, a b c}^{\Pi}(x, n) \equiv \sum_{j} \frac{j^{c} e^{-x(|j|+|j+n|-|n|)}}{|j|^{a-\frac{d-1}{2}}|j+n|^{b-\frac{d-1}{2}}} \kappa_{a-\frac{d}{2}}(|j| x) \kappa_{b-\frac{d}{2}}(|j+n| x)
\end{aligned}
$$

\section{D.1.1 First part $V_{I}^{\mathrm{f}}$ of Eq. (2.16)}

A generic form for the first part of $V^{\text {f }}$ from Eq. (2.16) (plus its $\{1 \leftrightarrow 2\}$ part) is now

$$
\begin{aligned}
V_{I}^{\mathrm{f}}\left(d, s_{1 \ldots 8}\right) & \equiv f_{P}^{\prime} \frac{\left(P_{0}\right)^{s_{6}}}{\left(P^{2}\right)^{s_{1}}}\left(\Pi_{s_{247}}-\Pi_{s_{247}}^{B}\right)\left(\Pi_{s_{358}}-\Pi_{s_{358}}^{B}\right) \\
& =\frac{T^{2 d}(2 \pi T)^{d+3+s_{678}-2 s_{12345}}}{2^{s_{12345}-1} \Gamma\left(s_{1}\right) \Gamma\left(s_{2}\right) \Gamma\left(s_{3}\right) \Gamma\left(s_{4}\right) \Gamma\left(s_{5}\right)} \sum_{n}^{\prime} \int_{0}^{\infty} \mathrm{d} x \int_{0}^{\infty} \mathrm{d} y e^{-|n|(x+y)} \times \\
& \times \frac{n^{s_{6}}|n|^{4-d-2 s_{1}}}{x^{d-s_{24}} y^{d-s_{35}}} h_{d, s_{1}}(|n| x,|n| y) \hat{f}_{d, s_{247}}^{\Pi-B}(x, n) \hat{f}_{d, s_{358}}^{\Pi-B}(y, n) \\
h_{d, s}(x, y) & =\frac{2^{s} \Gamma(s)}{\pi} \int_{0}^{\infty} \mathrm{d} z \frac{z^{3-d}}{\left(1+z^{2}\right)^{s}} j_{\frac{d-2}{2}}(x z) j_{\frac{d-2}{2}}(y z) \\
\hat{f}_{d, a b c}^{\Pi-B}(x, n) & =\sum_{j} \frac{j^{c} e^{-x(|j|+|j+n|-|n|)}}{|j|^{a-\frac{d-1}{2}}|j+n|^{b-\frac{d-1}{2}}} \kappa_{a-\frac{d}{2}}(|j| x) \kappa_{b-\frac{d}{2}}(|j+n| x)- \\
& -\frac{n^{c}}{|n|^{c}} \sum_{j=0}^{[c / 2]} \frac{\Gamma(a) \Gamma(b)(4 \pi)^{\frac{d+1}{2}}}{\Gamma\left(a+b-\frac{d+1}{2}-j\right)} \frac{g_{c, j}(a, b, d+1) \kappa_{a+b-d-j-1 / 2}(|n| x)}{(x / 2)^{j}|n|^{j+a+b-c-d}}
\end{aligned}
$$

In an expansion around odd $d$ and for integer indices $s_{i}$, the Bessel functions $\kappa$ reduce to (reverse) Bessel polynomials $\kappa_{s}(x)=\frac{\theta_{|s|-1 / 2}(x)}{x^{|s|-1 / 2}}$, such that $(\alpha=a+b-d-j-1 / 2)$

$$
\begin{aligned}
\hat{f}_{d, a b c}^{\Pi-B}(x, n) & \approx \sum_{k=0}^{\left|a-\frac{d}{2}\right|-\frac{1}{2}\left|b-\frac{d}{2}\right|-\frac{1}{2}} \sum_{\ell=0} \frac{\left(2\left|a-\frac{d}{2}\right|-1-k\right) !}{\left(\left|a-\frac{d}{2}\right|-\frac{1}{2}-k\right) ! k !} \frac{\left(2\left|b-\frac{d}{2}\right|-1-\ell\right) !}{\left(\left|b-\frac{d}{2}\right|-\frac{1}{2}-\ell\right) ! \ell !} \frac{s_{c, k-\left|a-\frac{d}{2}\right|-a+\frac{d}{2}, \ell-\left|b-\frac{d}{2}\right|-b+\frac{d}{2}}(x, n)}{(2 x)^{\left|a-\frac{d}{2}\right|+\left|b-\frac{d}{2}\right|-1-k-\ell}}- \\
& -\frac{n^{c}}{|n|^{c}} \frac{\Gamma(a) \Gamma(b)}{x^{d+c-a-b}} \sum_{j=0}^{[c / 2]} \frac{(4 \pi)^{\frac{d+1}{2}} g_{c, j}(a, b, d+1)}{\Gamma\left(\alpha+\frac{d}{2}\right)} \sum_{k=0}^{|\alpha|-\frac{1}{2}} \frac{(2|\alpha|-1-k) !}{\left(|\alpha|-\frac{1}{2}-k\right) ! k !} \frac{(|n| x)^{k-\alpha-|\alpha|+c-2 j}}{2^{|\alpha|-\frac{1}{2}-k-j}}+\mathcal{O}(\epsilon) \\
& \text { where } s_{c a b}(x, n)=\sum_{j} e^{-x(|j|+|j+n|-|n|)} j^{c}|j|^{a}|j+n|^{b}=\hat{s}_{c a b}(\operatorname{coth}(x), n), \\
& \hat{s}_{c a b}(y,-n)=(-1)^{c} \hat{s}_{c a b}(y, n), \quad \hat{s}_{0 a b}(y, \mathbb{Z})=\hat{s}_{0 b a}(y, \mathbb{Z}), \quad \hat{s}_{c a b}(y, 0)=\hat{s}_{c, a+b, 0}(y, 0) \\
& \hat{s}_{c_{\text {even }} a b}(y, n)=\hat{s}_{0, a+c, b}(y, n), \quad \hat{s}_{c_{\mathrm{odd}} a b}(y, n)=\hat{s}_{1, a+c-1, b}(y, n),
\end{aligned}
$$

\footnotetext{
${ }^{1}$ From Eqs. (3.9), (3.10), (3.11) we see that $\hat{f}_{d, a b c}^{X}(x,-n)=(-1)^{c} \hat{f}_{d, a b c}^{X}(x, n)$ for all $X \in\{\Pi, B, C, D\}$.
} 
or explicitly e.g.

$$
\begin{aligned}
& \hat{s}_{000}(c, n)=c+|n|, \quad \hat{s}_{010}(c, n)=\frac{1}{2}\left[c^{2}-1+n^{2}+|n| c\right], \\
& \hat{s}_{020}(c, n)=\frac{1}{6}\left[3 c\left(c^{2}-1+n^{2}\right)+|n|\left(3 c^{2}+2 n^{2}-2\right)\right], \\
& \hat{s}_{011}(c, n)=\frac{1}{6}\left[3 c\left(c^{2}-1\right)+|n|\left(3 c^{2}+n^{2}-4\right)\right], \\
& \hat{s}_{100}(c, n)=-\frac{n}{2}[c+|n|], \quad \hat{s}_{110}(c, n)=-\frac{n}{6}\left[3 c^{2}+2 n^{2}-2+|n| 3 c\right] .
\end{aligned}
$$

Note that $g_{c, j}(a, b, d+1) / \Gamma\left(\alpha+\frac{d}{2}\right)$ in Eq. (D.12) needs a proper $\epsilon \rightarrow 0$ limit. Meanwhile, the $j$ reduce to trigonometric functions, cf. Eq. (D.2), for which the integral in $h$ evaluates to exponentials times polynomials,

$$
h_{d, s}(x, y) \approx \frac{(-1)^{\frac{d-1}{2}}}{2 e^{x+y}} \theta(x-y)\left(p_{d, s}^{+}(x, y)+e^{2 y} p_{d, s}^{-}(x, y)\right)+(x \leftrightarrow y)+\mathcal{O}(\epsilon)
$$

where the polynomials $p^{ \pm}$contain even and odd parts $t^{ \pm}$of reverse Bessel polynomials

$$
\begin{aligned}
p_{d, s}^{ \pm}(x, y) & =\sum_{k=1}^{s}\left(\begin{array}{l}
s-1 \\
k-1
\end{array}\right) t_{1, k+\frac{d-5}{2}}(x)\left[y^{d-2} t_{0, s-k-\frac{d-1}{2}}(y) \pm y^{2 s-2 k} t_{0, k-s+\frac{d-3}{2}}(y)\right] \\
t_{b, n}(x) & =(2 n-1) t_{b, n-1}(x)+x^{2} t_{b, n-2}(x), \quad t_{b, 0}(x)=1, \quad t_{b, 1}(x)=1+b x \\
\Rightarrow & t_{b, n<0}(x)=x^{2 n+1}\left[b t_{-n-1}^{+}(x)+t_{-n-1}^{-}(x)\right], \quad t_{b, n \geq 0}(x)=t_{n}^{+}(x)+b t_{n}^{-}(x) \\
& t_{n}^{ \pm}(x)=\sum_{k=0}^{n} \frac{(2 n-k) !}{(n-k) ! k !} \frac{(2 x)^{k}}{2^{n+1}}\left(1 \pm(-1)^{k}\right)
\end{aligned}
$$

or explicitly e.g.

$$
\begin{aligned}
p_{3,3}^{ \pm}(x, y) & =(y \pm 1)(3+2 x) \pm\left(x+x^{2}+y^{2}\right) \\
p_{5,3}^{ \pm}(x, y) & =(y \pm 1)\left(15+15 x+6 x^{2}+x^{3}+y^{2}+x y^{2}\right) \pm \\
& \pm y^{2}\left(5+5 x+2 x^{2}\right) \\
p_{5,4}^{ \pm}(x, y) & =(y \pm 1)\left(105+105 x+45 x^{2}+10 x^{3}+x^{4}+10 y^{2}+10 x y^{2}+3 x^{2} y^{2}\right) \pm \\
& \pm y^{2}\left(35+35 x+15 x^{2}+3 x^{3}+y^{2}+x y^{2}\right) \\
p_{7,3}^{ \pm}(x, y) & =(y \pm 1)\left(315+315 x+135 x^{2}+30 x^{3}+3 x^{4}+30 y^{2}+30 x y^{2}+12 x^{2} y^{2}+2 x^{3} y^{2}\right) \pm \\
& \pm y^{2}\left(105+105 x+45 x^{2}+10 x^{3}+x^{4}+3 y^{2}+3 x y^{2}+x^{2} y^{2}\right)
\end{aligned}
$$

such that (using $\left.\hat{f}(-n)=(-1)^{c} \hat{f}(n)\right)$

$$
\begin{aligned}
& V_{I}^{\mathrm{f}}\left(d, s_{1 \ldots 8}\right)=\frac{T^{2}}{(4 \pi)^{\frac{3 d-1}{2}}} \sum_{n=1}^{\infty} \int_{0}^{\infty} \mathrm{d} x \int_{0}^{x} \mathrm{~d} y \frac{(4 \pi)^{\frac{3 d-1}{2}}}{\left(4 \pi^{2}\right)^{d-1}} \frac{(2 \pi T)^{3 d+1+s_{678}-2 s_{12345}} \pi^{\frac{d}{2}-1}(-1)^{\frac{d-1}{2}}}{2^{s_{12345}} \Gamma\left(s_{1}\right) \Gamma\left(s_{2}\right) \Gamma\left(s_{3}\right) \Gamma\left(s_{4}\right) \Gamma\left(s_{5}\right) \Gamma\left(\frac{d}{2}\right)} \times \\
& \times\left[1+(-1)^{s_{678}}\right] \frac{n^{s_{6}+4-d-2 s_{1}} e^{-2 n x}}{36(x y)^{2 d-2 s_{35}+s_{8}}}\left[p_{d, s_{1}}^{-}(n x, n y)+e^{-2 n y} p_{d, s_{1}}^{+}(n x, n y)\right] \times \\
& \times\left\{\frac{\tilde{f}_{d, s_{247}}^{\Pi-B}(x, n) \tilde{f}_{d, s_{358}}^{\Pi-B}(y, n)}{x^{2 s_{35}-2 s_{24}+s_{7}-s_{8}}}+(x \leftrightarrow y)\right\}+\mathcal{O}(\epsilon)
\end{aligned}
$$




$$
\text { where } \tilde{f}_{d, s_{247}}^{\Pi-B}(x, n)=6 x^{d+s_{7}-s_{24}} \hat{f}_{d, s_{247}}^{\Pi-B}(x, n) .
$$

For our sum-integral $\mathcal{M}_{3,-2}$, we need to evaluate five specific cases which follow from Eq. (D.27) and are all of similar structure, such as e.g. $\left(\operatorname{csch}^{2}(x)=\operatorname{coth}^{2}(x)-1\right)$

$$
\begin{aligned}
4 V_{I}^{\mathrm{f}}(3,31111,022) & =\frac{T^{2}}{(4 \pi)^{4}} \sum_{n=1}^{\infty} \int_{0}^{\infty} \mathrm{d} x \int_{0}^{x} \mathrm{~d} y \frac{e^{-2 n x}}{18 n^{5} x^{4} y^{4}}\left(3+3 n x+n^{2} x^{2}-n y-2 n(1+n x) y+\right. \\
& \left.+n^{2} y^{2}-e^{-2 n y}\left(3+3 n x+n^{2} x^{2}+n y+2 n(1+n x) y+n^{2} y^{2}\right)\right) \times \\
& \times\left(3+n x\left(3+3 n x-x^{2}\right)-3 x^{3}\left(n^{2} \operatorname{coth} x+(n+\operatorname{coth} x) \operatorname{csch}^{2} x\right)\right) \times \\
& \times\left(3+n y\left(3+3 n y-y^{2}\right)-3 y^{3}\left(n^{2} \operatorname{coth} y+(n+\operatorname{coth} y) \operatorname{csch}^{2} y\right)\right)+ \\
& +\mathcal{O}(\epsilon) .
\end{aligned}
$$

All sums are of the form $s_{a}(z>0)=\sum_{n=1}^{\infty} e^{-2 n z} / n^{a}=\operatorname{Li}_{a}\left(e^{-2 z}\right)$ resulting in $s_{a>1}(z)=$ $\mathrm{Li}_{a}\left(e^{-2 z}\right), s_{1}(z)=-\ln \left(1-e^{-2 z}\right)$ and $s_{a \leq 0}$ are polynomial of coth $z$. For the numerical evaluation of the various $V_{I}^{\mathrm{f}}$ we use Mathematica [23] $\left(\sum_{n} \rightarrow\right.$ thousands of $\mathrm{Li}_{j}$; then we utilize NIntegrate $[\ldots,\{\mathrm{x}, 0,1000\},\{\mathrm{y}, 0, \mathrm{x}\}$, MaxRecursion->100, WorkingPrecision->20]). As a result, weighting each piece by the prefactor with it contributes to $\mathcal{M}_{3,-2}$, we obtain

$$
\begin{aligned}
4 V_{I}^{\mathrm{f}}(3,31111,022) & =\frac{T^{2}}{(4 \pi)^{4}}[+0.01854774(1)+\mathcal{O}(\epsilon)], \\
12 V_{I}^{\mathrm{f}}(5,31122,011) & =\frac{T^{2}}{(4 \pi)^{7}}[+0.02392697(1)+\mathcal{O}(\epsilon)], \\
9 V_{I}^{\mathrm{f}}(5,41111,000) & =\frac{T^{2}}{(4 \pi)^{7}}[+0.00006691(1)+\mathcal{O}(\epsilon)], \\
6 V_{I}^{\mathrm{f}}(5,32111,000) & =\frac{T^{2}}{(4 \pi)^{7}}[+0.00100101(1)+\mathcal{O}(\epsilon)], \\
60 V_{I}^{\mathrm{f}}(7,33311,000) & =\frac{T^{2}}{(4 \pi)^{10}}[+0.01888983(1)+\mathcal{O}(\epsilon)] .
\end{aligned}
$$

\section{D.1.2 Second part $V_{I I}^{\mathrm{f}}$ of Eq. (2.16)}

The second part of $V^{\mathrm{f}}$ as in Eq. (2.16) is (this is only half of it; the other half is $247 \leftrightarrow 358$ )

$$
\begin{aligned}
& \hat{V}_{I I}^{\mathrm{f}}\left(d, s_{1 \ldots 8}\right) \equiv \mathcal{f}_{P}^{\prime} \frac{\left(P_{0}\right)^{s_{6}}}{\left(P^{2}\right)^{s_{1}}}\left(\Pi_{s_{247}}-\Pi_{s_{247}}^{C}\right)\left(\Pi_{s_{358}}^{B}-\Pi_{s_{358}}^{D}\right) \\
& =\frac{(2 \pi T)^{3 d+3+s_{678}-2 s_{12345}}}{\pi^{\frac{3(d+1)}{2}} 2^{2 s_{12345}+2 d}} \frac{\Gamma\left(\frac{1}{2}\right)}{\Gamma\left(\frac{d}{2}\right)} \frac{1+(-1)^{s_{678}}}{\Gamma\left(s_{2}\right) \Gamma\left(s_{4}\right)} \sum_{n=1}^{\infty} n^{d+3+s_{68}-2 s_{135}} \int_{0}^{\infty} \mathrm{d} x e^{-2 n x} \times \\
& \times x^{s_{24}-d} \hat{f}_{d, s_{247}}^{\Pi-C}(x, n) \tilde{f}_{d, s_{1358}}\left(n x, \frac{\alpha}{4 \pi^{2} n^{2}}, \epsilon\right), \\
& \tilde{f}_{d, s_{1358}}(x, y, \epsilon)=\sum_{j=0}^{\left[s_{8} / 2\right]} 2^{j} \frac{e^{x}}{\pi} \int_{0}^{\infty} \mathrm{d} z \frac{z j_{\frac{d}{2}-1}(x z)}{\left(\frac{1+z^{2}}{2}\right)^{s_{135}-\frac{d+1}{2}-j}}\left(1-\left(\frac{1+z^{2}}{y}\right)^{\epsilon}\right) \frac{1}{\epsilon} \epsilon(4 \pi)^{\frac{d+1}{2}} g_{s_{8}, j}\left(s_{3}, s_{5}, d+1\right)
\end{aligned}
$$




$$
\begin{aligned}
& \approx \ln \left(\frac{x y e^{\gamma}}{2}\right) \tilde{\ell}_{d, s_{1358}}(x)+e^{2 x} \operatorname{Ei}(-2 x) \tilde{\ell}_{d, s_{1358}}(-x)+\bar{\ell}_{d, s_{1358}}(x)+\mathcal{O}(\epsilon), \\
& \left(\begin{array}{l}
\tilde{\ell} \\
\bar{\ell}
\end{array}\right)_{d, s_{1358}}(x) \equiv \sum_{j=0}^{\left[s_{8} / 2\right]} 2^{j} \underbrace{\epsilon(4 \pi)^{\frac{d+1}{2}} g_{s_{8}, j}\left(s_{3}, s_{5}, d+1\right)}_{\mathbb{Q}+\mathcal{O}(\epsilon)} \underbrace{\left(\begin{array}{c}
\ell_{1} \\
\ell_{2}
\end{array}\right)\left(d, s_{135}-\frac{d+1}{2}-j, x\right)}_{\operatorname{poly}(\mathrm{x})}, \\
& \ell_{1}(d, s, x) \equiv \frac{x^{d-2}}{\Gamma(s)} \underbrace{e^{x} x^{s-\frac{d}{2}} \sqrt{\frac{\pi}{2}} K_{\frac{d}{2}-s}(x)}=\frac{x^{d-2}}{\Gamma(s)} \theta_{s-\frac{d+1}{2}}(x), \\
& \text { reverse Bessel poly } \\
& \theta_{n<0}(x)=x^{1+2 n} \theta_{-n-1}(x), \quad \theta_{n \geq 0}(x)=\sum_{k=0}^{n} \frac{(2 n-k) !}{(n-k) ! k !} \frac{(2 x)^{k}}{2^{n}}, \\
& \ell_{2}(d, s, x) \equiv \frac{e^{x}}{\pi} \int_{0}^{\infty} \mathrm{d} z \frac{z \sqrt{\frac{\pi}{2}}(x z)^{\frac{d}{2}-1} J_{\frac{d}{2}-1}(x z)}{\left(\frac{1+z^{2}}{2}\right)^{s}} \ln \frac{1}{1+z^{2}}- \\
& -\ln \left(\frac{x e^{\gamma}}{2}\right) \ell_{1}(d, s, x)-e^{2 x} \operatorname{Ei}(-2 x) \ell_{1}(d, s,-x) \text {. }
\end{aligned}
$$

From $\tilde{\ell}$ and $\bar{\ell}$, the cases that are useful for us read $\bar{\ell}_{\{3,3112\},\{5,3121\},\{5,4110\},\{5,3210\},\{5,3110\},\{7,3310\}}(x)=\left\{2 x^{2},-2 x^{3},-x^{3}, 3 x^{3},-2 x^{2}, x^{4}\right\} / 12$, $\bar{\ell}_{\{3,3112\},\{5,3121\},\{5,4110\},\{5,3210\},\{5,3110\},\{7,3310\}}(x)=-\left\{2 x^{2}-2 x,-2 x^{3},-x^{3}, 3 x^{3},-4 / 3 x^{2}, x^{4}\right\} / 8$.

The first part of $\hat{f}^{\Pi-C}=\hat{f}^{\Pi-B}-\hat{f}^{C-B}$ is defined in Eqs. (D.11) and (D.12) above, while the latter part is given by

$$
\begin{aligned}
\hat{f}_{d, a b c}^{C-B}(x, n) & =\frac{1+(-1)^{c}}{2} \zeta(2 a-c-d) \frac{|n|^{\frac{d-1}{2}} x^{\frac{d+1}{2}}}{2^{\frac{d-1}{2}} \pi^{\frac{1}{2}}} 2^{a} \Gamma\left(a-\frac{d}{2}\right) \frac{\kappa_{b-\frac{d}{2}}(|n| x)}{|n|^{b} x^{a}}+ \\
& +[-\operatorname{sign}(n)]^{c} \sum_{n=0}^{[c / 2]}\left(\begin{array}{c}
c \\
2 n
\end{array}\right) \zeta(2 b-2 n-d)|n|^{c-2 n} \frac{|n|^{\frac{d-1}{2}} x^{\frac{d+1}{2}}}{2^{\frac{d-1}{2}} \pi^{\frac{1}{2}}} 2^{b} \Gamma\left(b-\frac{d}{2}\right) \frac{\kappa_{a-\frac{d}{2}}(|n| x)}{|n|^{a} x^{b}} .
\end{aligned}
$$

We can therefore write the finite parts $V_{I I}^{\mathrm{f}}+\mathcal{O}(\epsilon)=\hat{V}_{I I}^{\mathrm{f}}\left(d, s_{1 \ldots 8}\right)+\hat{V}_{I I}^{\mathrm{f}}\left(d, s_{13254687}\right)+\mathcal{O}(\epsilon)$ as

$$
\begin{aligned}
4 V_{I I}^{\mathrm{f}}(3,31111,022) & =\frac{T^{2}}{(4 \pi)^{4}} \sum_{n=1}^{\infty} \int_{0}^{\infty} \mathrm{d} x \frac{2 x}{3} \hat{f}_{3,112}^{\Pi-C}(x, n)\left[\operatorname{Ei}(-2 n x)+e^{-2 n x} \ln \left(\frac{\alpha_{1} e^{\gamma-\frac{3}{2}}}{16 \pi^{2}} \frac{2 x}{n}\right)+\frac{3 e^{-2 n x}}{2 n x}\right], \\
12 V_{I I}^{\mathrm{f}}(5,31122,011) & =\frac{T^{2}}{(4 \pi)^{7}} \sum_{n=1}^{\infty} \int_{0}^{\infty} \mathrm{d} x \frac{4 x}{3} \hat{f}_{5,121}^{\Pi-C}(x, n)\left[\operatorname{Ei}(-2 n x)-e^{-2 n x} \ln \left(\frac{\alpha_{2} e^{\gamma-\frac{3}{2}}}{16 \pi^{2}} \frac{2 x}{n}\right)\right], \\
9 V_{I I}^{\mathrm{f}}(5,41111,000) & =\frac{T^{2}}{(4 \pi)^{7}} \sum_{n=1}^{\infty} \int_{0}^{\infty} \mathrm{d} x \frac{1}{n} \hat{f}_{5,110}^{\Pi-C}(x, n)\left[\operatorname{Ei}(-2 n x)-e^{-2 n x} \ln \left(\frac{\alpha_{3} e^{\gamma-\frac{3}{2}}}{16 \pi^{2}} \frac{2 x}{n}\right)\right], \\
6 V_{I I}^{\mathrm{f}}(5,32111,000) & =\frac{T^{2}}{(4 \pi)^{7}} \sum_{n=1}^{\infty} \int_{0}^{\infty} \mathrm{d} x \frac{-2}{3} \hat{f}_{5,210}^{\Pi-C}(x, n)\left[\operatorname{Ei}(-2 n x)+e^{-2 n x} \ln \left(\frac{\alpha_{4} e^{\gamma-1}}{16 \pi^{2}} \frac{2 x}{n}\right)\right]+
\end{aligned}
$$




$$
\begin{array}{r}
+\frac{T^{2}}{(4 \pi)^{7}} \sum_{n=1}^{\infty} \int_{0}^{\infty} \mathrm{d} x \frac{-1}{n} \hat{f}_{5,110}^{\Pi-C}(x, n)\left[\operatorname{Ei}(-2 n x)-e^{-2 n x} \ln \left(\frac{\alpha_{3} e^{\gamma-\frac{3}{2}}}{16 \pi^{2}} \frac{2 x}{n}\right)\right], \\
60 V_{I I}^{\mathrm{f}}(7,33311,000)=\frac{T^{2}}{(4 \pi)^{10}} \sum_{n=1}^{\infty} \int_{0}^{\infty} \mathrm{d} x \frac{2 x}{3} \hat{f}_{7,310}^{\Pi-C}(x, n)\left[\operatorname{Ei}(-2 n x)+e^{-2 n x} \ln \left(\frac{\alpha_{5} e^{\gamma-\frac{3}{2}}}{16 \pi^{2}} \frac{2 x}{n}\right)\right] .
\end{array}
$$

Note that line 3 and 5 cancel exactly (assuming they are finite). The reason is that $\Pi_{210}^{B}=$ $\frac{g_{00}(2,1, d+1)}{\left[P^{2}\right]^{3-\frac{d+1}{2}}}=\frac{3-d-1}{P^{2}} \frac{g_{00}(1,1, d+1)}{\left[P^{2}\right]^{2-\frac{d+1}{2}}}=\frac{2-d}{P^{2}} \Pi_{110}^{B}$ such that the relevant contributions to $\mathcal{M}_{3,-2}$ combine with a pre-factor of $\mathcal{O}(\epsilon)$ :

$$
\begin{aligned}
\mathcal{M}_{3,-2}^{\mathrm{nz}, \mathrm{f}} & \ni 2 d \mathcal{D}^{+}\left\{3 \hat{V}_{I I}^{\mathrm{f}}\left(41111,000, \alpha_{3}\right)+\hat{V}_{I I}^{\mathrm{f}}\left(31211,000, \alpha_{3}\right)\right\} \\
& =2 d \mathcal{D}^{+}\left\{3 \hat{V}_{I I}^{\mathrm{f}}\left(41111,000, \alpha_{3}\right)+(2-d) \hat{V}_{I I}^{\mathrm{f}}\left(41111,000, \alpha_{3}\right)\right\} \\
& =2 d \mathcal{D}^{+}\left\{(5-d) \hat{V}_{I I}^{\mathrm{f}}\left(41111,000, \alpha_{3}\right)\right\} \\
& =2 d(3-d) \mathcal{D}^{+} \hat{V}_{I I}^{\mathrm{f}}\left(41111,000, \alpha_{3}\right) .
\end{aligned}
$$

Thus, also the contributions from $V^{\mathrm{d}}$ should not contain $\alpha_{3}$ up to their constant terms, which is indeed the case, serving as a small check of our expressions. Furthermore, it appears convenient to choose $\alpha_{1}=\alpha_{2}=\alpha_{5}=16 \pi^{2} e^{3 / 2-\gamma}$ and $\alpha_{4}=16 \pi^{2} e^{1-\gamma}$, although the $\gamma$ could also remain in the log, see Eq. (D.52).

The various functions $f^{\Pi-C}$ above are (omitting the arguments $(x, n)$ on the lhs)

$$
\begin{aligned}
\hat{f}_{3,112}^{\Pi-C} & =\frac{1}{30 x^{3}}\left(x^{4}-15+5 n x\left(x^{2}-3\right)-5 n^{2} x^{2}\left(3+x^{2}\right)+15 x^{3}\left(n^{2} \operatorname{coth} x+(n+\operatorname{coth} x) \operatorname{csch}^{2} x\right)\right), \\
f_{5,121}^{\Pi-C} & =\frac{n}{6 x^{2}}\left(6+x\left(3 x+n\left(3+x^{2}\right)\right)-3 x \operatorname{coth} x(1+n x+x \operatorname{coth} x)\right), \\
f_{5,110}^{\Pi-C} & =-\frac{1}{90 x^{3}}\left(225+135 n x+15 n x^{3}+x^{4}+n x^{5}-\right. \\
& \left.-45 x\left(2(1+n x) \operatorname{coth} x+x(2+n x+x \operatorname{coth} x) \operatorname{csch}^{2} x\right)\right), \\
f_{5,210}^{\Pi-C} & =-\frac{1}{180 x^{2}}\left(270+120 x^{2}+x^{4}+30 n x\left(3+x^{2}\right)-90 x \operatorname{coth} x(2+n x+x \operatorname{coth} x)\right), \\
f_{7,310}^{\Pi-C} & =-\frac{1}{1890 x^{3}}\left(9450+x\left(3780 x+x^{5}+315 n^{2} x\left(3+x^{2}\right)+315 n\left(12+5 x^{2}\right)\right)-\right. \\
& \left.-945 x \operatorname{coth} x\left(6+3 n x+\left(-1+n^{2}\right) x^{2}+x \operatorname{coth} x(3+n x+x \operatorname{coth} x)\right)\right) .
\end{aligned}
$$

A quick numerical evaluation (summing up to $n_{\max }=10000$, and using Mathematica's NIntegrate [. . , , MaxRecursion->100, WorkingPrecision->60, AccuracyGoal->30], while estimating the error by fitting values $n \in[10000,60000]$ and extrapolating to infinity) of the (weighted) $V_{I I}^{\mathrm{f}}$ parts then produces (choosing all $\alpha_{i}=16 \pi^{2} e^{3 / 2-\gamma}$ )

$$
\begin{aligned}
4 V_{I I}^{\mathrm{f}}(3,31111,022) & =\frac{T^{2}}{(4 \pi)^{4}}[+0.00775440(1)+\mathcal{O}(\epsilon)], \\
12 V_{I I}^{\mathrm{f}}(5,31122,011) & =\frac{T^{2}}{(4 \pi)^{7}}[-0.00354681(1)+\mathcal{O}(\epsilon)],
\end{aligned}
$$




$$
\begin{aligned}
9 V_{I I}^{\mathrm{f}}(5,41111,000)+6 V_{I I}^{\mathrm{f}}(5,32111,000) & =\frac{T^{2}}{(4 \pi)^{7}}[+0.00295006(1)+\mathcal{O}(\epsilon)] \\
60 V_{I I}^{\mathrm{f}}(7,33311,000) & =\frac{T^{2}}{(4 \pi)^{10}}[-0.00503877(1)+\mathcal{O}(\epsilon)] .
\end{aligned}
$$

It might be possible to evaluate (some of) the $\hat{V}_{I I}^{\mathrm{f}}$ analytically. We have not put further effort into this, as the numerical values given above are fully sufficient for our purposes. Before leaving, let us record some integrals and some sums that might be useful in that respect:

$$
\begin{aligned}
& \int_{0}^{\infty} \mathrm{d} z e^{-z} z^{n}=\Gamma(n+1), \quad \int_{0}^{\infty} \mathrm{d} z \operatorname{Ei}(-z) z^{n}=\frac{\Gamma(n+1)}{n+1}, \quad \operatorname{Re}(n)>-1, \\
& \int_{0}^{\infty} \mathrm{d} z e^{-z} z^{n}(\gamma+\ln z)=\Gamma(n+1) \text { HarmonicNumber }(n), \quad \operatorname{Re}(n)>-1, \\
& \int_{0}^{\infty} \mathrm{d} z\left[e^{-z}(\gamma+\ln z)-\operatorname{Ei}(-z)\right] \frac{1}{z}=\zeta(2)=\frac{\pi^{2}}{6}, \\
& \sum_{n=1}^{\infty} \frac{\ln n}{n^{a}}=-\zeta^{\prime}(a), \quad \sum_{n=1}^{\infty} \frac{e^{-2 n x}}{n^{a}}=\operatorname{Li}_{a}\left(e^{-2 x}\right), \quad \sum_{n=1}^{\infty} \frac{e^{-2 n x} \ln n}{n^{a}}=-\partial_{a} \operatorname{Li}_{a}\left(e^{-2 x}\right) .
\end{aligned}
$$

One can get some analytic parts from looking at the piece $\sim \hat{f}_{5,110}^{C-B}(x, n)=\frac{x}{90}(1+|n| x)$ of

$$
\begin{aligned}
\hat{V}_{I I}^{\mathrm{f}}\left(5,41111,000, \alpha_{3}\right) & \ni \frac{T^{2}}{(4 \pi)^{7}} \sum_{n=1}^{\infty} \int_{0}^{\infty} \mathrm{d} x \frac{\hat{f}_{5,110}^{C-B}(x, n)}{18 n}\left[e^{-2 n x} \ln \left(\frac{\alpha_{3} e^{\gamma-\frac{3}{2}}}{16 \pi^{2}} \frac{2 x}{n}\right)-\operatorname{Ei}(-2 n x)\right] \\
& =\frac{T^{2}}{(4 \pi)^{7}} \frac{1}{18} \sum_{n=1}^{\infty}\{\frac{1}{108 n^{3}} \underbrace{\frac{3}{20} \int_{0}^{\infty} \mathrm{d} z z(2+z)\left[e^{-z} \ln \left(z e^{\gamma}\right)-\operatorname{Ei}(-z)\right]}_{=1}- \\
& -\frac{\ln n}{90 n^{3}} \underbrace{\frac{1}{4} \int_{0}^{\infty} \mathrm{d} z z(2+z) e^{-z}}_{=1}\}=\frac{T^{2}}{(4 \pi)^{7}} \frac{1}{18^{2}}\left(\frac{\zeta(3)}{6}+\frac{\zeta^{\prime}(3)}{5}\right),
\end{aligned}
$$

where in the second line we have chosen $\alpha_{3}=16 \pi^{2} e^{3 / 2}$, and which is confirmed by the corresponding analytic expression for the $\left(\Pi^{C}-\Pi^{B}\right)$ piece of $\hat{V}^{\mathrm{d}}$, which can be extracted by considering only the $I \cdot I$ parts of Eq. (3.15).

\section{D.1.3 Summing up}

Summing up Eqs. (D.30)-(D.34) and Eqs. (D.44)-(D.47), we obtain the numerical coefficient of Eq. (3.19) as

$$
n_{1} \approx+0.0645513(1)
$$

\section{D.2 Contribution to $\mathcal{M}_{3,-2}$ from finite parts $V^{\text {z,f }}$ of zero-modes}

Here, we discuss the evaluation of the finite terms given in Eq. (4.16) of the main text. 
Working again in coordinate space, we need the Fourier transform for $\Pi^{A}$ of Eq. (4.8). Being the sum of two terms, its first part can be read off from the last line of Eq. (D.12), while in the notation of Eq. (D.6) with $P_{0}=0$ its second part (let us label it $E$ here) reads

$$
\hat{f}_{d, a b 0}^{E}(2 \pi T r, 0)=\frac{\Gamma(d / 2-a) \Gamma(d / 2-b)}{\Gamma^{2}(1 / 2)}\left(\frac{2 \pi T r}{2}\right)^{a+b+1-d},
$$

whereas below we also need the special case $\hat{f}_{3,222}^{E}(x, 0)=1$.

What we need to evaluate is the first line of Eq. (4.7) for which, using Eq. (D.2) we obtain

$$
\begin{aligned}
S^{\mathrm{f}}\left(d ; s_{1 \ldots 5} ; s_{7}, s_{8}\right) & =\sum_{P} \frac{\delta_{P_{0}}}{\left[P^{2}\right]^{s_{1}}}\left(\Pi_{s_{247}}-\Pi_{s_{247}}^{A}\right)\left(\Pi_{s_{358}}-\Pi_{s_{358}}^{A}\right) \\
& =\frac{(2 \pi T)^{3 d+3+s_{78}-2 s_{12345}} 2^{1-2 d-s_{12345}}}{\Gamma\left(s_{1}\right) \Gamma\left(s_{2}\right) \Gamma\left(s_{3}\right) \Gamma\left(s_{4}\right) \Gamma\left(s_{5}\right) \Gamma(d / 2) \pi^{1+3 d / 2}} \int_{0}^{\infty} \mathrm{d} x \int_{0}^{x} \mathrm{~d} y \hat{h}_{d, s_{1}}(x, y) \times \\
& \times\left[x^{s_{24}-d} y^{s_{35}-d} \hat{f}_{s_{247}-A}^{\Pi-A}(x, 0) \hat{f}_{s_{358}}^{\Pi-A}(y, 0)+\{x \leftrightarrow y\}\right]
\end{aligned}
$$

with $\hat{h}_{d, s}(x, y)=\frac{\Gamma(d / 2-s) y^{d-2}}{2^{s} \Gamma(d / 2)} x_{2 s-2}^{2} F_{1}\left(d / 2-s, 1-s, d / 2, y^{2} / x^{2}\right) \quad(d>2 s>0)$,

and special cases $\hat{h}_{\{31,51,52,72\}}(x, y)=\left\{y, \frac{y^{3}}{3}, \frac{y^{3}}{3} \frac{5 x^{2}+y^{2}}{5}, \frac{y^{5}}{15} \frac{7 x^{2}-3 y^{2}}{7}\right\}$.

The function $\hat{h}$ in fact originates from

$$
\frac{2^{s} \Gamma(s)}{\pi} \int_{0}^{\infty} \mathrm{d} z z^{3-d-2 s} j_{d / 2-1}(x z) j_{d / 2-1}(y z)=\theta(x-y) \hat{h}_{d, s}(x, y)+\theta(y-x) \hat{h}_{d, s}(y, x),
$$

which corresponds to the overall $\int_{\mathbf{p}}$ of Eq. (D.55) after performing the angular integrals.

To get an explicit expression of $f^{\Pi-A}$, we simply refer to Eq. (D.12), taken at $n=0$ and where we only have to insert the theta function of Eq. (4.8) into the second line, as well as Eq. (D.54) above, again multiplied by the theta function. For the specific cases that we are interested in, we get the compact expressions

$$
\begin{aligned}
\frac{4}{5} S_{0110}^{\mathrm{f}}(3,12121,22) & =\frac{T^{2}}{(4 \pi)^{4}} \int_{0}^{\infty} \mathrm{d} x \int_{0}^{x} \mathrm{~d} y \frac{1}{5 x^{4} y^{3}}\left[f_{1}(x, y)+\{x \leftrightarrow y\}\right] \\
f_{1}(x, y) & =x^{5}(\operatorname{coth} x-1)\left(y^{3} \operatorname{coth}(y) \operatorname{csch}^{2} y-1\right) \\
\frac{4}{5} S_{1010}^{\mathrm{f}}(3,12211,22) & =\frac{T^{2}}{(4 \pi)^{4}} \int_{0}^{\infty} \mathrm{d} x \int_{0}^{x} \mathrm{~d} y \frac{1}{5 x^{2} y}\left(1-x^{2} \operatorname{csch}^{2} x\right)\left(1-y^{2} \operatorname{csch}^{2} y\right) \\
& =\frac{T^{2}}{(4 \pi)^{4}} \int_{0}^{\infty} \mathrm{d} x \frac{1}{5 x^{2}}\left(1-x^{2} \operatorname{csch}^{2} x\right)(\ln (x \operatorname{csch} x)+x \operatorname{coth} x-1), \\
-\frac{6}{5} S_{0110}^{\mathrm{f}}(5,22121,00) & =\frac{T^{2}}{(4 \pi)^{7}} \int_{0}^{\infty} \mathrm{d} x \int_{0}^{x} \mathrm{~d} y \frac{-2\left(5 x^{2}+y^{2}\right)}{75 x^{6} y^{3}}\left[f_{3}(x, y)+\{x \leftrightarrow y\}\right] \\
f_{3}(x, y) & =x^{5}(\operatorname{coth} x-1)\left(2 y \operatorname{coth} y-5+y^{2}(2+y \operatorname{coth} y) \operatorname{csch}^{2} y\right) \\
-\frac{12}{5} S_{0110}^{\mathrm{f}}(5,12221,00) & =\frac{T^{2}}{(4 \pi)^{7}} \int_{0}^{\infty} \mathrm{d} x \int_{0}^{x} \mathrm{~d} y \frac{-4}{15 x^{4} y}\left[f_{4}(x, y)+\{x \leftrightarrow y\}\right]
\end{aligned}
$$




$$
\begin{aligned}
f_{4}(x, y) & =x^{3}(\operatorname{coth} x-1)\left(-3+y \operatorname{csch}^{2}(y)(y+\sinh (2 y))\right) \\
96 S_{0110}^{\mathrm{f}}(7,23321,00) & =\frac{T^{2}}{(4 \pi)^{10}} \int_{0}^{\infty} \mathrm{d} x \int_{0}^{x} \mathrm{~d} y \frac{4\left(7 x^{2}-3 y^{2}\right)}{525 x^{6} y}\left[f_{5}(x, y)+\{x \leftrightarrow y\}\right] \\
f_{5}(x, y) & =x^{3}(\operatorname{coth} x-1)(2+x+x \operatorname{coth} x)\left(6 y \operatorname{coth} y-10+y^{2}(3+y \operatorname{coth} y) \operatorname{csch}^{2} y\right) \\
84 S_{0110}^{\mathrm{f}}(7,23222,00) & =\frac{T^{2}}{(4 \pi)^{10}} \int_{0}^{\infty} \mathrm{d} x \int_{0}^{x} \mathrm{~d} y \frac{7 x^{2}-3 y^{2}}{75 x^{6} y}\left[f_{6}(x, y)+\{x \leftrightarrow y\}\right] \\
f_{6}(x, y) & =x^{3}(\operatorname{coth} x-1)(2+x+x \operatorname{coth} x)\left(2 y \operatorname{coth} y-5+y^{2}(2+y \operatorname{coth} y) \operatorname{csch}^{2} y\right) .
\end{aligned}
$$

The $y$-integration can be explicitly performed in most (all but the last two) cases, giving a result containing zetas and (poly)logarithms, whereas the remaining $x$-integration can be approximated numerically, to give

$$
\begin{aligned}
\frac{4}{5} S^{\mathrm{f}}(3,12121,22) & \approx \frac{T^{2}}{(4 \pi)^{4}}[-0.01005114745(1)+\mathcal{O}(\epsilon)], \\
\frac{4}{5} S^{\mathrm{f}}(3,12211,22) & \approx \frac{T^{2}}{(4 \pi)^{4}}[+0.15213227462(1)+\mathcal{O}(\epsilon)], \\
-\frac{6}{5} S^{\mathrm{f}}(5,22121,00) & \approx \frac{T^{2}}{(4 \pi)^{7}}[-0.00891125885(1)+\mathcal{O}(\epsilon)], \\
-\frac{12}{5} S^{\mathrm{f}}(5,12221,00) & \approx \frac{T^{2}}{(4 \pi)^{7}}[-0.10673211253(1)+\mathcal{O}(\epsilon)], \\
96 S^{\mathrm{f}}(7,23321,00) & \approx \frac{T^{2}}{(4 \pi)^{10}}[+0.20021689747(1)+\mathcal{O}(\epsilon)], \\
84 S^{\mathrm{f}}(7,23222,00) & \approx \frac{T^{2}}{(4 \pi)^{10}}[+0.02318282360(1)+\mathcal{O}(\epsilon)] .
\end{aligned}
$$

\section{D.2.1 Summing up}

Summing up Eqs. (D.64)-(D.69) we obtain the numerical coefficient of Eq. (4.16) as

$$
n_{2} \approx+0.24983747686(1)
$$

\section{E Cross-checks}

Using the generic formulae for spectacles-type sum-integrals as derived in the main text, we can check two particular cases, $\mathcal{M}_{1,0}$ and $V_{2}$, against the previously known results from the literature.

First, let us check $\mathcal{M}_{1,0}$ of Sec. 2 in [11]: In the language developed here,

$$
\mathcal{M}_{1,0}=V(11111,000)=V^{\mathrm{f}}+V^{\mathrm{d}}+V^{\mathrm{z}}=\mathcal{B}+2 \mathcal{C}+V^{\mathrm{d}}(11111,000)+\mathcal{A}+S^{\mathrm{d}}(11111,00),
$$

with $\{\mathcal{A}, \mathcal{B}, \mathcal{C}\}$ as defined in [11] and whose expansion (using $p_{31}^{ \pm}(x, y)= \pm 1$ as well as Eqs. (3.14), (4.9) and $L(111,00, d)=0$ ) exactly reproduces Eqs. (2.15)-(2.17) of Ref. [11].

Second, let us check $V_{2}$ of [12]: In the language developed here,

$$
V_{2}=V(21111,002)=V^{\mathrm{f}}+V^{\mathrm{d}}+V^{\mathrm{z}}=\mathcal{V}_{2}+\mathcal{V}_{4}+\mathcal{V}_{6}+V^{\mathrm{d}}(21111,002)+S(21111,02),
$$

where IBP on $S(21111,02)$ gives the reduction

$$
\mathcal{V}_{1}=S(21111,02)=\frac{1}{d-6}\left\{S(12111,02)+S(12111,20)-I_{2}^{0} A(211,2)-I_{2}^{2} A(211,0)\right\}
$$


for which

$$
\begin{aligned}
& S(12111,02)=\mathcal{V}_{1 a}+S^{\mathrm{d}}(12111,02), \\
& S(12111,20)=\mathcal{V}_{1 b}+S^{\mathrm{d}}(12111,20) .
\end{aligned}
$$

Collecting and expanding (using $p_{32}^{ \pm}(x, y)=y \pm(x+1)$; Eqs. (3.14), (4.9); as well as $L(111,00, d)=0 ; L(211,20, d)=0$; and $L(211,02, d)$ from Eq. (B.7)), one reproduces Eqs. (4.1)-(4.2) of Ref. [12].

\section{References}

[1] K. G. Chetyrkin and F. V. Tkachov, Integration by Parts: The Algorithm to Calculate beta Functions in 4 Loops, Nucl. Phys. B 192 (1981) 159; F. V. Tkachov, A Theorem on Analytical Calculability of Four Loop Renormalization Group Functions, Phys. Lett. B 100 (1981) 65;

S. Laporta, High precision calculation of multiloop Feynman integrals by difference equations, Int. J. Mod. Phys. A 15 (2000) 5087 [hep-ph/0102033]; M. Argeri and P. Mastrolia, Feynman Diagrams and Differential Equations, Int. J. Mod. Phys. A 22 (2007) 4375 [arXiv:0707.4037];

E. Remiddi and J. A. M. Vermaseren, Harmonic polylogarithms, Int. J. Mod. Phys. A 15 (2000) 725 [hep-ph/9905237]; J. A. M. Vermaseren, Harmonic sums, Mellin transforms and integrals, Int. J. Mod. Phys. A 14 (1999) 2037 [hep-ph/9806280]; S. Moch, P. Uwer and S. Weinzierl, Nested sums, expansion of transcendental functions and multiscale multiloop integrals, J. Math. Phys. 43 (2002) 3363 [hep-ph/0110083]; G. Heinrich, Sector Decomposition, Int. J. Mod. Phys. A 23 (2008) 1457 [arXiv:0803.4177].

[2] P. B. Arnold and C. X. Zhai, The Three loop free energy for pure gauge QCD, Phys. Rev. D 50 (1994) 7603 [hep-ph/9408276].

[3] E. Braaten and A. Nieto, Free energy of QCD at high temperature, Phys. Rev. D 53 (1996) 3421 [hep-ph/9510408].

[4] K. Kajantie, M. Laine, K. Rummukainen and Y. Schröder, How to resum long distance contributions to the QCD pressure?, Phys. Rev. Lett. 86 (2001) 10 [hep-ph/0007109]; K. Kajantie, M. Laine, K. Rummukainen and Y. Schröder, The Pressure of hot QCD up to $g^{6}$ $\ln (1 / g)$, Phys. Rev. D 67 (2003) 105008 [hep-ph/0211321].

[5] F. Di Renzo, M. Laine, V. Miccio, Y. Schröder and C. Torrero, The Leading non-perturbative coefficient in the weak-coupling expansion of hot QCD pressure, JHEP 0607 (2006) 026 [hep-ph/0605042].

[6] M. Laine and Y. Schröder, Quark mass thresholds in QCD thermodynamics, Phys. Rev. D 73 (2006) 085009 [hep-ph/0603048].

[7] Y. Schröder, Loops for Hot QCD, Nucl. Phys. Proc. Suppl. 183B (2008) 296 [arXiv:0807.0500].

[8] J. Möller and Y. Schröder, Open problems in hot QCD, Nucl. Phys. Proc. Suppl. 205-206 (2010) 218 [arXiv:1007.1223].

[9] J. Möller and Y. Schröder, Three-loop matching coefficients for hot QCD: Reduction and gauge independence, arXiv:1207.1309; J. Möller and Y. Schröder, Dimensionally reduced QCD at high temperature, Prog. Part. Nucl. Phys. 67 (2012) 168; J. Möller, Algorithmic approach to finite-temperature QCD, Diploma Thesis, University of Bielefeld (2009). 
[10] M. Nishimura and Y. Schröder, IBP methods at finite temperature, arXiv:1207.4042.

[11] Y. Schröder, A fresh look on three-loop sum-integrals, arXiv:1207.5666.

[12] I. Ghişoiu and Y. Schröder, A new three-loop sum-integral of mass dimension two, arXiv:1207.6214.

[13] P. H. Ginsparg, First Order and Second Order Phase Transitions in Gauge Theories at Finite Temperature, Nucl. Phys. B 170 (1980) 388; T. Appelquist and R. D. Pisarski, High-Temperature Yang-Mills Theories and Three-Dimensional Quantum Chromodynamics, Phys. Rev. D 23 (1981) 2305; K. Kajantie, M. Laine, K. Rummukainen and M. E. Shaposhnikov, Generic rules for high temperature dimensional reduction and their application to the standard model, Nucl. Phys. B 458 (1996) 90 [hep-ph/9508379].

[14] E. Braaten and A. Nieto, Effective field theory approach to high temperature thermodynamics, Phys. Rev. D 51 (1995) 6990 [hep-ph/9501375].

[15] I. Ghişoiu, J. Möller and Y. Schröder, in preparation; see also Strong and Electroweak Matter, Swansea, UK, 2012, http://pyweb.swan.ac.uk/sewm/sewmweb/posters/ghisoiu.pdf.

[16] A. Gynther, M. Laine, Y. Schröder, C. Torrero and A. Vuorinen, Four-loop pressure of massless $O(N)$ scalar field theory, JHEP 0704 (2007) 094 [hep-ph/0703307].

[17] J. O. Andersen, L. Kyllingstad and L. E. Leganger, Pressure to order $g^{8} \log g$ of massless $\phi^{4}$ theory at weak coupling, JHEP 0908 (2009) 066 [arXiv:0903.4596].

[18] A. Gynther, A. Kurkela and A. Vuorinen, The $N_{f}^{3} g^{6}$ term in the pressure of hot QCD, Phys. Rev. D 80 (2009) 096002 [arXiv:0909.3521].

[19] O. V. Tarasov, Connection between Feynman integrals having different values of the space-time dimension, Phys. Rev. D 54 (1996) 6479 [hep-th/9606018]; O. V. Tarasov, Generalized recurrence relations for two loop propagator integrals with arbitrary masses, Nucl. Phys. B 502 (1997) 455 [hep-ph/9703319].

[20] J. O. Andersen and L. Kyllingstad, Four-loop Screened Perturbation Theory, Phys. Rev. D 78 (2008) 076008 [arXiv:0805.4478].

[21] C. Bogner and S. Weinzierl, Feynman graph polynomials, Int. J. Mod. Phys. A 25 (2010) 2585 [arXiv:1002.3458].

[22] R. M. Pirsig, Zen and the Art of Motorcycle Maintenance, William Morrow \& Co. (1974).

[23] Wolfram Research, Inc., Mathematica, Version 8.0, Champaign, IL (2012). 